\title{
Lévy Distributions for One-Dimensional Analysis of the Bose-Einstein Correlations
}

\author{
V. A. Okorokov \\ National Research Nuclear University MEPhI (Moscow Engineering Physics Institute), Kashirskoe Shosse 31, Moscow 115409, Russia \\ Correspondence should be addressed to V. A. Okorokov; vaokorokov@mephi.ru
}

Received 16 May 2016; Revised 25 October 2016; Accepted 17 November 2016; Published 2 January 2017

Academic Editor: Frank Filthaut

Copyright (C) 2017 V. A. Okorokov. This is an open access article distributed under the Creative Commons Attribution License, which permits unrestricted use, distribution, and reproduction in any medium, provided the original work is properly cited. The publication of this article was funded by SCOAP $^{3}$.

\begin{abstract}
A general study of relations between the parameters of two centrally symmetric Lévy distributions, often used for one-dimensional investigation of Bose-Einstein correlations, is given for the first time. These relations of the strength of correlations and of the radius of the emission region take into account possible various finite ranges of the Lorentz invariant four-momentum difference for two centrally symmetric Lévy distributions. In particular, special cases of the relations are investigated for Cauchy and normal (Gaussian) distributions. The mathematical formalism is verified using the recent measurements given that a generalized centrally symmetric Lévy distribution is used. The reasonable agreement is observed between estimations and experimental results for all available types of strong interaction processes and collision energies.
\end{abstract}

\section{Introduction}

Correlations between two identical bosons, called Bose-Einstein correlations (BEC), are a well-known phenomenon in high-energy and nuclear physics. These correlations play an important role in the studies of multiparticle production and soft physics. Constructive interference affects the joint probability for the emission of a pair of identical bosons with four-momentum $p_{1}$ and $p_{2}$. Experimentally, the onedimensional BEC effect is observed as an enhancement at low values of the Lorentz invariant quantity $q=\sqrt{-\left(p_{1}-p_{2}\right)^{2}} \geq$ 0 in the two-particle correlation function (CF):

$$
\mathbf{C}_{2}(q)=\frac{\rho(q)}{\rho_{\text {ref }}(q)} .
$$

Here $\rho$ is the two-particle density function and $\rho_{\text {ref }}$ is a reference two-particle density function that by construction is expected to include no BEC. The detailed shape analysis of the peak of CF is an important topic on theoretical and experimental points of view because this shape carries information about the possible features of space-time structure of particle source $[1,2]$. For instance, the detail investigations have to do for shape of correlation peak in modern experiments with high statistics for verification of hypothesis of possible selfaffine fractal-like geometry of emission region $[3,4]$. The BEC effect in one dimension is usually described by a few-parameter function for which several different functional forms have been proposed. The power-law parametrization $\mathbf{C}_{2}(q) \sim$ $q^{-\beta}$ is the important signature for fractal-like source extending over a large volume $[5,6]$. The quite reasonable fit is achieved with this parametrization of two-pion CF in various types of multiparticle production processes [1]. But unfortunately power-law fits are absent for high-statistics modern experimental data so far. On the other hand the stable (on Lévy) distributions [7] are one of the most promising tools for studies of fractal-like space-time extent of emission region. These distributions are a rich class of probability distributions that allow skewness and heavy tails and have many important physical applications. As shown in $[3,4]$ the subclass of nonisotropic centrally symmetric Lévy distributions $[8,9]$ is most useful for studies of Bose-Einstein CF. Therefore this subclass of centrally symmetric Lévy distributions is considered regarding of BEC measurements in the present paper. 
For low-dimensional (1D) analysis the centrally symmetric Lévy distribution results in the most general parametrization of the experimental Bose-Einstein CF

$$
\begin{aligned}
& \mathrm{C}_{2}(q) \propto 1+\Omega(\alpha, \lambda, z) \\
& \quad \Omega(\alpha, \lambda, z) \equiv \lambda \exp \left(-|z|^{\alpha}\right), z \equiv q R .
\end{aligned}
$$

Here $\lambda$ is the strength of correlations called also chaoticity, $R$ is the $1 \mathrm{D} \mathrm{BEC}$ radius, and $0<\alpha \leq 2$ is the Lévy index called also index of stability. As known for a static source with no final state interactions $[10,11]$, there is the relation $\mathbf{C}_{2}(q) \propto$ $|\tilde{f}(q)|^{2}, \tilde{f}(q)=\int d x \exp ($ iqx $) f(x)$; that is, Bose-Einstein CF $\mathbf{C}_{2}(q)$ measures the absolute value squared Fourier transformed source density in the coordinate space $f(x), \int d x f(x)=$ 1 , called also coordinate-space distribution function of the particle emission points. The various experiments use the different forms of (2) which correspond to the various hypotheses with regard of $f(x)$. For example, most of the earliest experiments with particle beams used the specific case of (2) at $\alpha=2$; the Gaussian parametrization corresponded to the normal (Gaussian) distribution function $f_{G}(x)=\left(2 \pi R_{G}^{2}\right)^{-1 / 2} \exp \left[-\left(x-x_{0}\right)^{2} / 2 R_{G}^{2}\right]$, where the Gaussian scale parameter is $R_{G}^{2}=\left\langle x^{2}\right\rangle-x_{0}^{2}$, the standard deviation; then another specific case of (2) at $\alpha=1$ is used widely, especially, for particle (not nuclear) collisions. Equation (2) at $\alpha=1$ is called exponential parametrization for Bose-Einstein $\mathrm{CF} \mathrm{C}_{2}(q)$ and it corresponds to the Cauchy (Lorentzian) distribution function $f_{C}(x)=\pi^{-1} R_{C} /\left[R_{C}^{2}+\left(x-x_{0}\right)^{2}\right]$ with scale parameter $R_{C}[12,13]$. Furthermore the recent studies at the LHC [14-17] demonstrate that general view of (2) allows the reasonable description of experimental CF, particular for proton-proton $(p+p)$ collisions but for centrally symmetric Lévy distribution with $\alpha \in(0 ; 2), \alpha \neq 1$ the corresponding source density in coordinate space $f(x)$ can be written analytically for $\alpha=3 / 2,2 / 3,1 / 2,1 / 3$ only [9]. It is often difficult to compare results from different experiments because of the many different data analysis methods [11], in particular due to various parameterizations for $1 \mathrm{D}$ Bose-Einstein CF $\mathrm{C}_{2}(q)$. Therefore the derivation of the relations between the sets of BEC parameters for two centrally symmetric Lévy distributions is the important task for correct comparison of the results from different experiments, creation of the global kinematic (energy, pair transverse momentum, etc.) dependencies of BEC parameters, and so on. Such studies are important for investigations of common features of softstage dynamics in various multiparticle production processes as well as for equation of state (EoS) of strongly interacting matter, in particular, search for phase transition to the quarkgluon deconfined matter. It would be noted the study of energy dependence of pion BEC parameters in heavy ion collisions [18] was one of the main causes and drivers for hypothesis of cross-over transition from strongly coupled quark-gluon phase to hadronic one at Relativistic Heavy Ion Collider (RHIC) energies $\sqrt{s_{N N}} \sim 100 \mathrm{GeV}$. Furthermore some results for deconfinement in small system $[19,20]$ indicate remarkable similarity of both the bulk and the thermodynamic properties of strongly interacting matter created in high-energy $p+p / \bar{p}+p$ and $A+A$ collisions.
The BEC can provide new knowledge about collectivity and possible creation of droplets of quark-gluon matter in small system collisions. For these studies the correct comparison can be crucially important for BEC parameters in various multiparticle processes for wide energy range. But as mentioned above Bose-Einstein $\mathrm{CF}_{2}(q)$ is often described by different view of (2) depending on type of reaction, collision energy, and features of experimental analysis. Therefore the study of centrally symmetric Lévy distributions and search for relations between parameters for corresponding CF has scientific interest for physics of strong interactions.

The paper is organized as follows. In Section 2, mathematical formalism is described for case of two general view centrally symmetric Lévy distributions. Dependencies of desired 1D BEC observables on $q$ and $\alpha$ are studied for a priori known parameters for second centrally symmetric Lévy distributions. Section 3 is devoted to the detailed discussion of specific case of these distributions, namely, Cauchy and Gaussian ones most used in experimental investigations of $1 \mathrm{D} \mathrm{CF} \mathrm{C}_{2}(q)$. Database of experimental results for set of $1 \mathrm{D}$ BEC parameters $\{\lambda, R\}$ for charged pion source in strong interaction processes is created within the framework of the paper in order to verify the mathematical formalism. Section 4 demonstrates the comparison between the estimations calculated for 1D BEC parameters with help of mathematical formalism under discussion and available experimental results for various reactions and in wide energy range (it should be noted that in Sections 3 and 4 the 1D BEC parameters are supplied with the subindexes according to the names of corresponding source distribution function; namely, " $L$ " is for the general view of centrally symmetric Lévy distribution, " $C$ " is for the Cauchy source distribution function, and " $G$ " is for Gaussian one. Otherwise the notations $\left\{\lambda_{E}, R_{E}\right\}$ are often used in papers for second case due to relation between Cauchy distribution for $f(x)$ and exponential parametrization for Bose-Einstein $\mathrm{CF}_{2}(q)$ discussed above. As a consequence the mathematically rigorous terminology is used over full manuscript: the term "Cauchy distribution" corresponds to the source function in coordinate space $f_{C}(x)$ and the term "exponential function/parametrization" is used for the related parametrization of correlation function $\mathrm{C}_{2, E}(q)$; for the case of arbitrary $0<\alpha<2, \alpha \neq 1$, the term "centrally symmetric Lévy" is suitable for both the source function in coordinate space $f_{L}(x)$ and the parametrization of correlation function $\mathbf{C}_{2, L}(q)$; the similar situation is for $\alpha=2$ : the term "Gaussian" is applicable for both the source function in coordinate space $f_{G}(x)$ and the corresponding parametrization of correlation function $\mathbf{C}_{2, G}(q)$ ). In Section 5 some final remarks are presented. The experimental database is shown in Appendix for 1D BEC parameters.

\section{Relations between BEC Parameters in General Case}

Let some experimental $\mathrm{CF} \mathrm{C}_{2}(q)$ be described by two parameterizations (2) with $\Omega_{1} \equiv \Omega\left(\alpha_{1}, \lambda_{1}, z_{1}\right)$ and $\Omega_{2} \equiv$ $\Omega\left(\alpha_{2}, \lambda_{2}, z_{2}\right)$. Then relations between parameters of $\Omega_{1}$ and $\Omega_{2}$ can be deduced on the basis that both parameterizations 
describe one experimental $\mathrm{CF}_{2}(q)$, that is one sample of experimental points (in general the approximations of $\mathbf{C}_{2}(q)$ are characterized by different qualities for various parameterizations (2) with $\Omega_{1}$ and $\Omega_{2}$. The influence of this difference is not studied in present work and can be considered as separate task). Thus one can assume that the areas under fit curves for two parameterizations (2) with $\Omega_{1}$ and $\Omega_{2}$ are approximately equal to each other as well as the first moments of the corresponding centrally symmetric Lévy distributions.

2.1. Mathematical Formalism. The relations between two sets of parameters $\left\{\lambda_{1}, R_{1}\right\}$ and $\left\{\lambda_{2}, R_{2}\right\}$ of the particle source can be derived from the following system of equations:

$$
\begin{aligned}
& S_{1}=S_{2}, \quad \forall i=1,2: S_{i} \equiv \int_{J_{i}} \Omega\left(\alpha_{i}, \lambda_{i}, z_{i}\right) d q ; \\
& \langle q\rangle_{1}=\langle q\rangle_{2}, \\
& \quad \forall i=1,2:\langle q\rangle_{i} \equiv S_{i}^{-1} \int_{J_{i}} q \Omega\left(\alpha_{i}, \lambda_{i}, z_{i}\right) d q .
\end{aligned}
$$

The first equation (3a) corresponds to the equality of the areas under fit curves and the (3b) is the equality of the first moments of the $\Omega\left(\alpha_{i}, \lambda_{i}, z_{i}\right)$ distributions (as discussed above the approximate equalities are expected for areas and first moments in general case. This softer condition is enough for applicability of the formalism suggested in the paper. But the exactly equal signs are used in (3a) and (3b) as well as in the text below in order to get the mathematically correct forms for the systems of equations). System (3a) and (3b) contains the equations allowing the estimation of unknown strength of correlations and 1D BEC radius based on the available values of these parameters but it supposes the Lévy indexes $\alpha_{i}$ are known a priori for both parameterizations $\Omega_{i}, i=1,2$. In equations (3a) and (3b) the integrals are taken over full fit ranges $J_{i}, i=1,2$, for corresponding parameterizations with $\Omega_{i}$ of experimental CF. It should be noted that in general case (i) the ranges of integration can be different for parameterizations with $\Omega_{1}$ and $\Omega_{2}$; (ii) the full fit range can be the set of subranges due to possible exception of some intervals of the relative 4-momentum (regions of resonance contributions, etc.), that is, $\forall i=1,2: J_{i}=\bigcup_{k=1}^{N_{i}} J_{i}^{k} \equiv \bigcup_{k=1}^{N_{i}}\left[q_{k, \min }, q_{k, \max }\right]$ and consequently for all types of integrals and $\forall i=1,2$ in system (3a) and (3b): $\int_{J_{i}} \rightarrow \sum_{k=1}^{N_{i}} \int_{J_{i}^{k}}$. But usually the fit ranges $J_{i}$ are identical for both $\Omega_{i}, i=1,2$, in experimental studies (see, e.g., [15]). In general case of the centrally symmetric Lévy distributions and finite fit ranges the system equations under consideration cannot be solved analytically. The numerical procedure should be used in order to get the relations between two sets of parameters $\left\{\lambda_{1}, R_{1}\right\}$ and $\left\{\lambda_{2}, R_{2}\right\}$ of the particle source in this case. Without loss of generality $\Omega_{1}$ and $\alpha_{2}$ are considered as known and values of BEC parameters $\left\{\lambda_{2}, R_{2}\right\}$ are supposed as desired below. Then for specific case of semi-infinite ranges for integration $\forall i=1,2: J_{i}=$ $[0 ; \infty)$ system (3a) and (3b) can be solved analytically and one can derive the following ultimate relations between two sets $\left\{\lambda_{1}, R_{1}\right\}$ and $\left\{\lambda_{2}, R_{2}\right\}$ of BEC parameters for corresponding centrally symmetric Lévy parameterizations with $\Omega_{i}, i=1,2$

$$
\begin{aligned}
& \lambda_{1}^{u}=\lambda_{2}\left[\alpha_{1} \Gamma\left(2 \alpha_{1}^{-1}\right) \Gamma^{2}\left(\alpha_{2}^{-1}\right)\right] \\
& \cdot {\left[\alpha_{2} \Gamma^{2}\left(\alpha_{1}^{-1}\right) \Gamma\left(2 \alpha_{2}^{-1}\right)\right]^{-1} ; } \\
& R_{1}^{u}=R_{2}\left[\Gamma\left(2 \alpha_{1}^{-1}\right) \Gamma\left(\alpha_{2}^{-1}\right)\right]\left[\Gamma\left(\alpha_{1}^{-1}\right) \Gamma\left(2 \alpha_{2}^{-1}\right)\right]^{-1}
\end{aligned}
$$

and vice versa. Here $\Gamma(x)=\int_{0}^{\infty} t^{x-1} \exp (-t) d t, \operatorname{Re} x>0$ is the gamma function.

In the point of view of data analysis the absence of the general analytic relations between $\left\{\lambda_{1}, R_{1}\right\}$ and $\left\{\lambda_{2}, R_{2}\right\}$ leads to the following approach for estimations of the errors of the unknown parameters. Without the loss of generality let us suppose that the values are known for set of parameters $\left\{\alpha_{1}, \lambda_{1}, R_{1}\right\}$ with its errors $\left\{\Delta^{ \pm} \alpha_{1}, \Delta^{ \pm} \lambda_{1}, \Delta^{ \pm} R_{1}\right\}$ for centrally symmetric Lévy parametrization with $\Omega_{1}$ as well as for $\alpha_{2}$ with $\Delta^{ \pm} \alpha_{2}$ for parametrization with $\Omega_{2}$. The two sets of values for unknown BEC parameters $\left\{\lambda_{2}, R_{2}\right\}$ can be obtained with the help of suitable system of equations: the input values $\left\{\alpha_{1}+\right.$ $\left.\Delta^{+} \alpha_{1}, \lambda_{1}+\Delta^{+} \lambda_{1}, R_{1}+\Delta^{+} R_{1}\right\}$ and $\alpha_{2}+\Delta^{+} \alpha_{2}$ produce the output set $\left\{\lambda_{2}^{+}, R_{2}^{+}\right\}$and $\left\{\alpha_{1}-\Delta^{-} \alpha_{1}, \lambda_{1}-\Delta^{-} \lambda_{1}, R_{1}-\Delta^{-} R_{1}, \alpha_{2}-\Delta^{-} \alpha_{2}\right\} \rightarrow$ $\left\{\lambda_{2}^{-}, R_{2}^{-}\right\}$. Then the error estimations for set $\left\{\lambda_{2}, R_{2}\right\}$ of BEC parameters for parametrization with $\Omega_{2}$ can be calculated as follows:

$$
\Delta^{ \pm} Y_{2}=\left|Y_{2}^{ \pm}-Y_{2}\right|, \quad Y_{2} \equiv \lambda_{2}, R_{2} .
$$

One can use the errors (5) which are asymmetric in general case or make the averaging of up and low uncertainties and then to use the symmetric errors $\Delta Y_{2}=\left(\Delta^{+} Y_{2}+\Delta^{-} Y_{2}\right) / 2$.

2.2. Dependencies on $q$ and $\alpha$ Variables. Figure 1 shows dependence of $1 \mathrm{D}$ BEC radius $(\mathrm{a}, \mathrm{b})$ and strength of correlations (c, d) for centrally symmetric Lévy parametrization $\Omega_{1}$ with known $\alpha_{1}=1.5$ on low $q_{1}$ (a, c) and high $q_{2}$ (b, d) limits of integration in system (3a) and (3b) for set $\left\{\alpha_{2}, \lambda_{2}, R_{2}\right\}=$ $\{0.5,0.5,1.5 \mathrm{fm}\}$ for $\Omega_{2}$. The solid lines correspond to the indicated values of $q_{2}$ in $\mathrm{GeV} / \mathrm{c}$ for $q_{1}$-dependence (a, c) and to shown values of the $q_{1}$ in $\mathrm{GeV} / \mathrm{c}$ for $q_{2}$-dependence (b, d). Values of BEC parameters $\lambda_{1}$ and $R_{1}$ depend strongly on the fixed second limit of integration $\left(q_{2(1)}\right)$ for both the $q_{1}$ - (Figures $1(\mathrm{a})$ and $1(\mathrm{c})$ ) and the $q_{2}$-dependence (Figures $1(\mathrm{~b})$ and $1(\mathrm{~d})$ ). The dashed lines correspond to the results from system (3a) and (3b) with $q_{2} \rightarrow \infty$ for $q_{1}$-dependence (Figures 1(a) and 1(c)) and with $q_{1}=0$ for $q_{2}$-dependence (Figures 1(b) and 1(d)). As seen the curves for general case of (3a) and (3b) coincide with dashed lines at $q_{2}=10 \mathrm{GeV} / \mathrm{c}$ for $q_{1}$-dependence (Figures $1(\mathrm{a})$ and $1(\mathrm{c})$ ) and at $q_{1}=10^{-3} \mathrm{GeV} / \mathrm{c}$ for $q_{2}$-dependence (Figures 1(b) and 1(d)). These values for $q_{1}$ and especially for $q_{2}$ are far from the corresponding limit in modern experimental CF. Therefore one should use system (3a) and (3b) with finite limits in an integrations in the case of experimentally available $q$-ranges for two parameterizations with $\Omega_{1}$ and $\Omega_{2}$. The thin dotted lines demonstrate the ultimate levels for $R_{1}$ (Figures 1(a) and 1(b)) and $\lambda_{1}$ (Figures $1(c)$ and $1(d)$ ) calculated with (4a) and (4b) for given values 


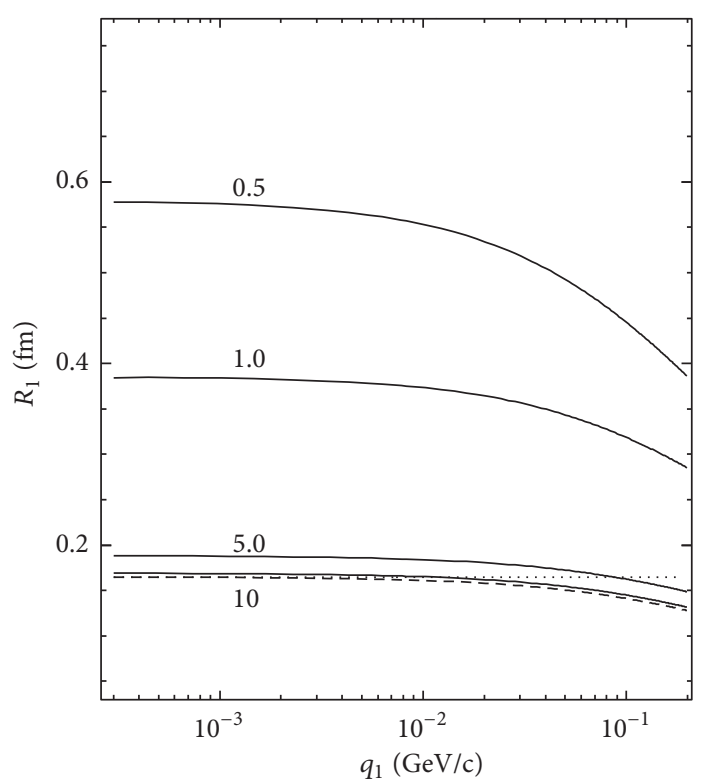

(a)

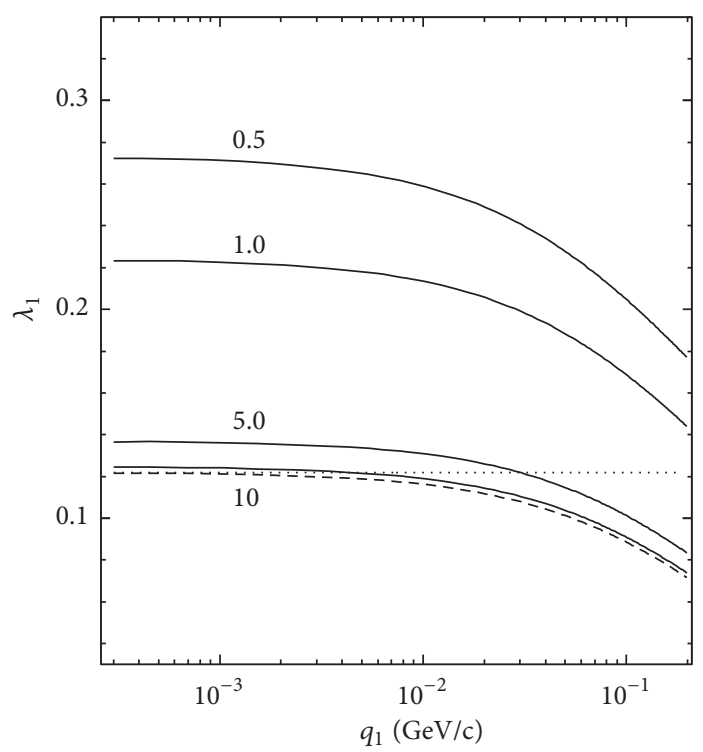

(c)



(b)

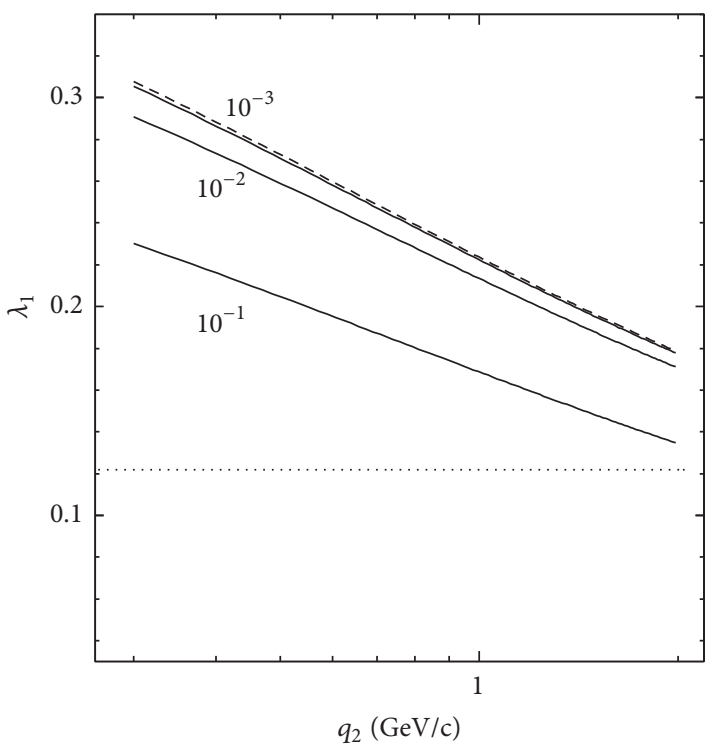

(d)

FIGURE 1: Dependence of 1D BEC radius (a, b) and strength of correlations (c, d) for centrally symmetric Lévy parametrization with $\alpha_{1}=1.5$ on low $q_{1}(\mathrm{a}, \mathrm{c})$ and high $q_{2}(\mathrm{~b}, \mathrm{~d})$ limits of integration in the system of (3a) and (3b) for fixed parameter values for second centrally symmetric Lévy parametrization: $\alpha_{2}=0.5, \lambda_{2}=0.5$, and $R_{2}=1.5 \mathrm{fm}$. The solid lines correspond to the indicated values of the $q_{2}$ for $q_{1}$-dependence (a, c) and to shown values of the $q_{1}$ for $q_{2}$-dependence (b, d). The dashed lines correspond to the calculations with $q_{2} \rightarrow \infty$ for $q_{1}$-dependence $(\mathrm{a}, \mathrm{c})$ and with $q_{1}=0$ for $q_{2}$-dependence (b, d). The thin dotted lines are the ultimate levels for $R_{1}(\mathrm{a}, \mathrm{b})$ and $\lambda_{1}(\mathrm{c}, \mathrm{d})$ calculated with (4a) and (4b) for given values of $\alpha_{1}$ and the set of parameters $\left\{\alpha_{2}, \lambda_{2}, R_{2}\right\}$ for second Lévy parametrization.

of $\alpha_{1}$ and the set of parameters $\left\{\alpha_{2}, \lambda_{2}, R_{2}\right\}$ for second Lévy parametrization $\Omega_{2}$. One can use the simple relations (4a) and (4b) for calculation $\left\{\lambda_{1}, R_{1}\right\}$ at $q_{1} \lesssim 10^{-2} \mathrm{GeV} / \mathrm{c}$ and $q_{2} \gtrsim 10 \mathrm{GeV} / \mathrm{c}$ (Figures $1(\mathrm{a})$ and $1(\mathrm{~b})$ ) but as expected the values of BEC parameters $\left\{\lambda_{1}, R_{1}\right\}$ are far from the ultimate levels at any $q_{1}$ for $q_{2}$-dependence in the range $q_{2} \leq 2 \mathrm{GeV} / \mathrm{c}$ is considered in Figures 1(b) and 1(d).

In Figure 2 dependence of $1 \mathrm{D}$ BEC radius $(a, b)$ and strength of correlations (c, d) is demonstrated for centrally symmetric Lévy parametrization with $\Omega_{1}$ on $\alpha_{1}$ at fixed values of $\alpha_{2}(\mathrm{a}, \mathrm{c})$ and on $\alpha_{2}$ at fixed values of $\alpha_{1}(\mathrm{~b}, \mathrm{~d})$ for given limits of integration in the system of (3a) and (3b) $q_{1}=0.02 \mathrm{GeV} / \mathrm{c}$, $q_{2}=2.0 \mathrm{GeV} / \mathrm{c}$ and for certain values of the BEC parameters for second centrally symmetric Lévy parametrization with $\Omega_{2}: \lambda_{2}=0.5$ and $R_{2}=1.5 \mathrm{fm}$. The solid lines correspond to the indicated values of the $\alpha_{2}$ for $\alpha_{1}$-dependence (a, c) and to shown values of the $\alpha_{1}$ for $\alpha_{2}$-dependence (b, d). The values for limits of integration $q_{1}$ and $q_{2}$ in system (3a) and (3b) 


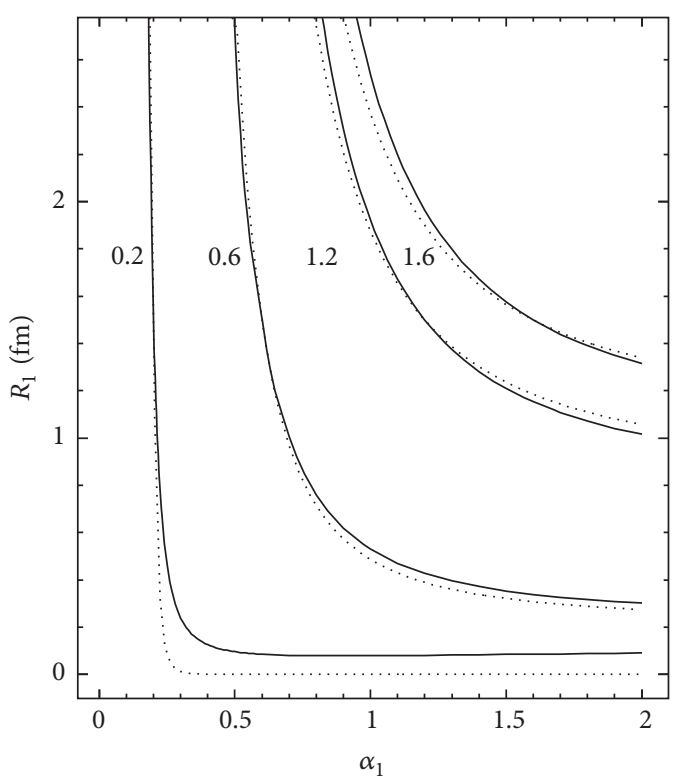

(a)

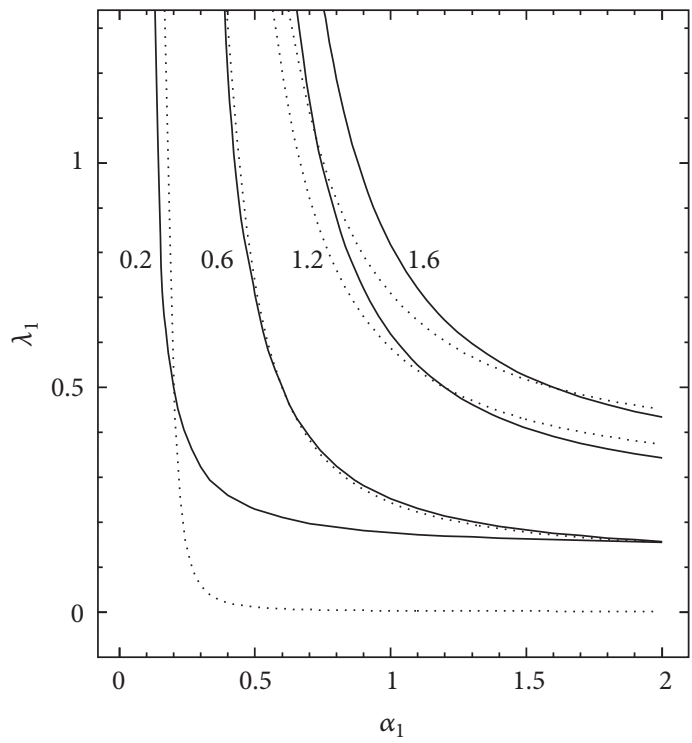

(c)

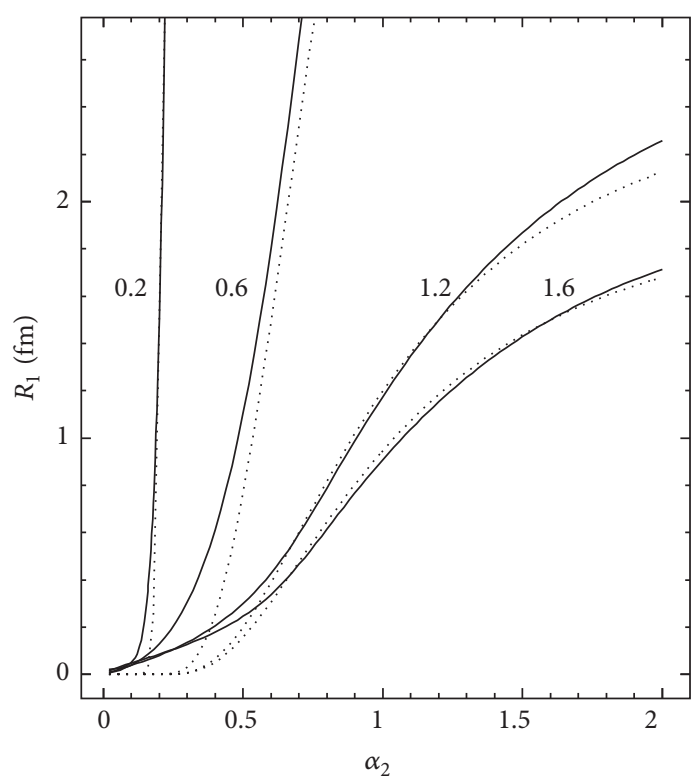

(b)

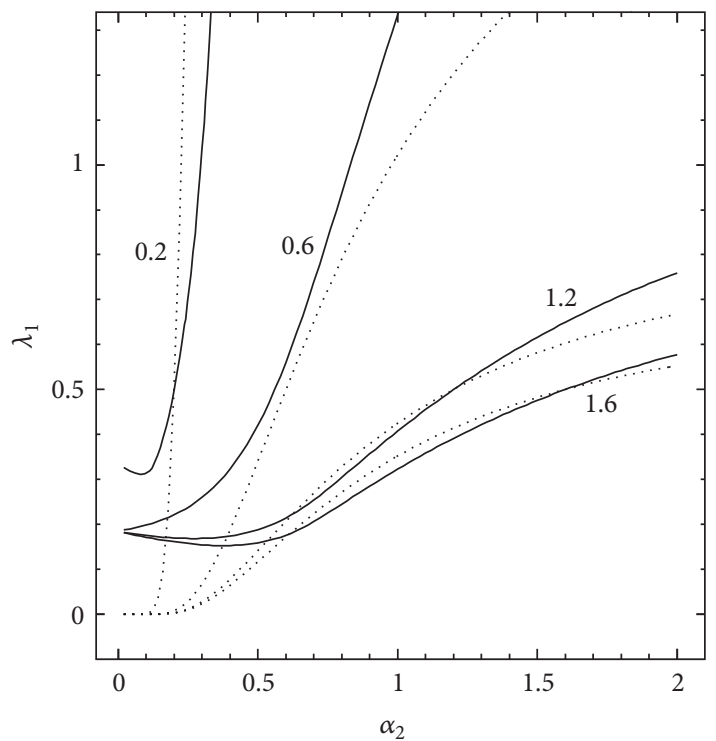

(d)

Figure 2: Dependence of 1D BEC radius $(\mathrm{a}, \mathrm{b})$ and strength of correlations $(\mathrm{c}, \mathrm{d})$ for centrally symmetric Lévy parametrization on $\alpha_{1}$ at fixed values of $\alpha_{2}(\mathrm{a}, \mathrm{c})$ and on $\alpha_{2}$ at fixed values of $\alpha_{1}(\mathrm{~b}, \mathrm{~d})$ for given limits of integration in the system of $(3 \mathrm{a})$ and $(3 \mathrm{~b}) q_{1}=0.02 \mathrm{GeV} / \mathrm{c}$, $q_{2}=2.0 \mathrm{GeV} / \mathrm{c}$ and for fixed values of the BEC parameters for second centrally symmetric Lévy parametrization: $\lambda_{2}=0.5$ and $R_{2}=1.5 \mathrm{fm}$. The solid lines correspond to the indicated values of the $\alpha_{2}$ for $\alpha_{1}$-dependence (a, c) and to shown values of the $\alpha_{1}$ for $\alpha_{2}$-dependence (b, d). The dotted lines are the ultimate cases for $R_{1}(\mathrm{a}, \mathrm{b})$ and $\lambda_{1}(\mathrm{c}, \mathrm{d})$ calculated with (4a) and (4b) for given values of $\alpha_{1}(\mathrm{a}, \mathrm{c})$ or $\alpha_{2}(\mathrm{~b}, \mathrm{~d})$ and the set of BEC parameters $\left\{\lambda_{2}, R_{2}\right\}$ for second Lévy parametrization.

are similar to those used in modern experiments. As seen dependencies of both BEC parameters on $\alpha_{i}, i=1,2$, at fixed another Lévy index $\left.\alpha_{j}\right|_{j \neq i}, j=1,2$, change very fast at small value $\alpha_{j}=0.2$ in narrow range $\alpha_{i} \simeq \alpha_{j}$. Such behavior is observed for both the results from system (3a) and (3b) and the estimations for $R_{1}$ (Figures 2(a) and 2(b)) and $\lambda_{1}$ (Figures 2(c) and 2(d)) calculated with (4a) and (4b) for semi-infinite ranges for integration and shown by dotted lines. The dependence $R_{1}\left(\alpha_{1}\right)$ shown in Figure 2(a) closes to the analytic one calculated with help of (4b) and presented by dotted line for any $\alpha_{2}$ under study with exception of the small value $\alpha_{2}=0.2$. For last case the agreement is obtained in very narrow range $\alpha_{1} \approx 0.2$ between results from system (3a) and (3b) and (4b). This feature maps clearly in corresponding dependencies $R_{2}\left(\alpha_{2}\right)$ shown in Figure 2(b) for $\alpha_{1}=0.2$. For large $\alpha_{1}>1.0$ solid and dotted lines are close to each other in the range $\alpha_{2}>0.5$ but agreement is poor significantly between results from system (3a) and ( $3 \mathrm{~b}$ ) and ( $4 \mathrm{~b})$ for $\alpha_{1}=0.6$ especially in 
domain $\alpha_{2}<0.5$ (Figure 2(b)) taking into account the sharp behavior for corresponding dependence $R_{1}\left(\alpha_{2}\right)$. In general the behavior of dependencies of $\lambda_{1}$ on $\alpha_{1}$ (Figure 2(c)) and $\alpha_{2}$ (Figure 2(d)) is similar to the corresponding dependencies of $1 \mathrm{D}$ BEC radius $R_{1}$. But the agreement is poor usually between the results deduced from system (3a) and (3b) and shown by the solid lines and the estimations calculated based on (4a) and presented by the dotted lines. Therefore for values of limits of integration $q_{1,2}$ under consideration the approximate relations (4a) and (4b) should be used carefully for experimental analysis of dependencies on Lévy indexes and last equations can produce the reasonable estimations for BEC parameters for ranges $\forall i=1,2: \alpha_{i} \gtrsim 1$ only.

As seen the mathematical formalism described above and the results in Figures 1 and 2 are quantitative basis for choice of the applying of general equations (3a) and (3b) or ultimate relations (4a) and (4b) in data analysis for given experiment. Thus the method suggested in the paper is helpful for experimental and phenomenological studies of BEC in various processes at different parameterizations of $\mathrm{CF}_{2}(q)$ corresponding to the centrally symmetric Lévy source distributions.

\section{Relations between BEC Parameters in Specific Cases}

As seen in Figure 2 both dependencies of the 1D BEC radius $R_{1}$ ( $\left.\mathrm{a}, \mathrm{b}\right)$ and the strength of correlations $\lambda_{1}$ (c, d) on Lévy indexes $\alpha_{i}, i=1,2$, show the weaker changing in the domain $\forall i=1,2: \alpha_{i} \gtrsim 1$ in comparison with the range of small values of Lévy indexes. The region $\forall i=1,2: \alpha_{i} \gtrsim 1$ includes in particular the specific cases of Cauchy and Gaussian distributions for which corresponding parameterizations of BoseEinstein $\mathrm{CF}_{2}(q)$ with $\alpha=1$ and $\alpha=2$ are used mostly for experimental studies. Therefore these certain views of $\Omega_{i}$ are studied in detail below. Let $\Omega_{1} \equiv \Omega_{G}=\Omega\left(2, \lambda_{G}, R_{G}\right)$ for Gaussian parametrization (2) and $\Omega_{2} \equiv \Omega_{C}=\Omega\left(1, \lambda_{C}, R_{C}\right)$ for $1 \mathrm{D}$ approximation of experimental CF $\mathrm{C}_{2}(q)$ by exponential function.

3.1. Mathematical Formalism. Relations (4a) and (4b) are valid at any values of indexes of stability $0<\alpha_{i} \leq 2$ in two centrally symmetric Lévy parameterizations with $\Omega_{i}, i=1,2$. If without loss of generality $\left\{\lambda_{C}, R_{C}\right\}$ are considered as a priori known and values of Gaussian BEC parameters $\left\{\lambda_{G}, R_{G}\right\}$ are supposed as desired then as expected (4a) and (4b) result in the ultimate relations:

$$
\begin{aligned}
\lambda_{G}^{u} & =\frac{2 \lambda_{C}}{\pi} ; \\
R_{G}^{u} & =\frac{R_{C}}{\sqrt{\pi}}
\end{aligned}
$$

and vice versa. Relation (6a) is derived in [21] for the first time while formula (6b) for $1 \mathrm{D}$ BEC radii is well-known.
The following relations can be obtained from general system (3a) and (3b) for finite ranges of integrations and specific values $\alpha_{1}=2$ and $\alpha_{2}=1$ :

$$
\begin{aligned}
& \frac{\lambda_{G} \sqrt{\pi}}{2 R_{G}} \sum_{j=1}^{N_{G}}\left[\operatorname{erf}\left(z_{2, j, G}\right)-\operatorname{erf}\left(z_{1, j, G}\right)\right]=\frac{\lambda_{C}}{R_{C}} \\
& \cdot \sum_{i=1}^{N_{C}}\left[\exp \left(-z_{1, i, C}\right)-\exp \left(-z_{2, i, C}\right)\right] ; \\
& \frac{1}{R_{G} \sqrt{\pi}} \frac{\sum_{j=1}^{N_{G}}\left[\exp \left(-z_{1, j, G}^{2}\right)-\exp \left(-z_{2, j, G}^{2}\right)\right]}{\sum_{j=1}^{N_{G}}\left[\operatorname{erf}\left(z_{2, j, G}\right)-\operatorname{erf}\left(z_{1, j, G}\right)\right]}=\frac{1}{R_{C}} \\
& \cdot \frac{\sum_{i=1}^{N_{C}}\left[\exp \left(-z_{1, i, C}\right)\left(1+z_{1, i, C}\right)-\exp \left(-z_{2, i, C}\right)\left(1+z_{2, i, C}\right)\right]}{\sum_{i=1}^{N_{C}}\left[\exp \left(-z_{1, i, C}\right)-\exp \left(-z_{2, i, C}\right)\right]} .
\end{aligned}
$$

Here $\operatorname{erf}(x)=(2 / \sqrt{\pi}) \int_{0}^{x} \exp \left(-t^{2}\right) d t$ is the error integral and $z_{1(2), i, C(G)} \equiv q_{1(2), i, C(G)} R_{C(G)}$ are the limits for integration over corresponding subranges for Cauchy (Gaussian) distribution. The detailed study of all available experimental results in strong interaction processes for 1D parametrization (2) with $\alpha_{1}=2$ and $\alpha_{2}=1$ for experimental CF $\mathbf{C}_{2}(q)$ shows the following: (i) the ranges of integration for both the exponential and the Gaussian functions are equal; (ii) usually, the range of integration is not divided into subranges; in any case, such division is identical for both functions under consideration and maximum value of $N_{C(G)}$ is equal 2 for experimental analyses. Thus the general statement with regard of identity of integration ranges for $\Omega_{1}$ and $\Omega_{2}$ is quite confirmed for case of Cauchy and Gaussian distributions and $N_{C(G)} \equiv N$ in system (7a) and (7b).

Further simplification for the system of (7a) and (7b) depends on features of certain experiment; direction of calculations $\Omega_{C} \rightleftarrows \Omega_{G}$, that is, what kind of a set of BEC parameters of the two, $\left\{\lambda_{C}, R_{C}\right\}$ and $\left\{\lambda_{G}, R_{G}\right\}$, it is regarded as a priori known and which set is supposed as desired; and requirement on the accuracy level. For available experimental data for BEC of charged pion pairs produced in strong interaction processes (i) the accuracy for $1 \mathrm{D}$ BEC radius is better usually than that for $\lambda$ parameter and (ii) the accuracy for 1D BEC parameters in modern experiments is not better than $\sim 10^{-3}$ so far. Thus one can assume the conservative accuracy level $\varepsilon=5 \times 10^{-4}$. At present the most complex case with $N=2$ is for analyses of proton-proton collisions at some LHC energies only $[15,22]$. For this case all contributions are negligible from the subrange of $q$ values larger than the region of the influence of meson resonances excluded from the experimental fits; that is, all terms for $i=j=2$ can be omitted at given $\varepsilon$ and direction of calculation from a priori known Cauchy parameters to desired Gaussian parameters $\left\{\lambda_{C}, R_{C}\right\} \rightarrow\left\{\lambda_{G}, R_{G}\right\}$. But the statement is wrong for opposite direction of calculation from a priori known Gaussian parameters to desired Cauchy parameters $\left\{\lambda_{G}, R_{G}\right\} \rightarrow\left\{\lambda_{C}, R_{C}\right\}$ at 
$\varepsilon=5 \times 10^{-4}$. Therefore the sum can be omitted in system (7a) and $(7 \mathrm{~b})$ and equations can be rewritten as follows:

$$
\begin{aligned}
& \frac{\lambda_{G}}{R_{G} \sqrt{\pi}}\left[\operatorname{erf}\left(z_{2, G}\right)-\operatorname{erf}\left(z_{1, G}\right)\right] \\
& =\frac{2 \lambda_{C}}{\pi R_{C}}\left[\exp \left(-z_{1, C}\right)-\exp \left(-z_{2, C}\right)\right] ; \\
& \frac{1}{R_{G} \sqrt{\pi}} \frac{\exp \left(-z_{1, G}^{2}\right)-\exp \left(-z_{2, G}^{2}\right)}{\operatorname{erf}\left(z_{2, G}\right)-\operatorname{erf}\left(z_{1, G}\right)} \\
& =\frac{1}{R_{C}} \frac{\exp \left(-z_{1, C}\right)\left(1+z_{1, C}\right)-\exp \left(-z_{2, C}\right)\left(1+z_{2, C}\right)}{\exp \left(-z_{1, C}\right)-\exp \left(-z_{2, C}\right)}
\end{aligned}
$$

for all experiments in the case of estimation of unknown BEC parameters for Gaussian function based on the a priori known set of corresponding parameters for exponential function and for all experiments with exception of $p+p$ collisions at $\sqrt{s_{N N}}=0.9,2.36$, and $7 \mathrm{TeV}[15,22]$ in the case of inverse problem. Accounting the relation $R_{G} \leq R_{C} / \sqrt{\pi}$ and properties of the functions $\exp \left(-x^{2}\right), \operatorname{erf}(x)$ allows us to simplify (8a) and (8b) to system

$$
\begin{aligned}
& \frac{\lambda_{G}}{R_{G} \sqrt{\pi}} \operatorname{erfc}\left(z_{1, G}\right) \\
& =\frac{2 \lambda_{C}}{\pi R_{C}}\left[\exp \left(-z_{1, C}\right)-\exp \left(-z_{2, C}\right)\right] \\
& \frac{1}{R_{G} \sqrt{\pi}} \frac{\exp \left(-z_{1, G}^{2}\right)}{\operatorname{erfc}\left(z_{1, G}\right)} \\
& =\frac{1}{R_{C}} \frac{\exp \left(-z_{1, C}\right)\left(1+z_{1, C}\right)-\exp \left(-z_{2, C}\right)\left(1+z_{2, C}\right)}{\exp \left(-z_{1, C}\right)-\exp \left(-z_{2, C}\right)},
\end{aligned}
$$

where $\operatorname{erfc}(x)=1-\operatorname{erf}(x)$. The last system of equations is valid for remain set of experimental results with exception of the WA98 data [23] at given $\varepsilon$. Also the transition from system (8a) and (8b) to simpler equations (9a) and (9b) is not valid for CPLEAR data [24] for direction of calculation from a priori known Gaussian parameters to desired Cauchy parameters $\left\{\lambda_{G}, R_{G}\right\} \rightarrow\left\{\lambda_{C}, R_{C}\right\}$ at $\varepsilon=5 \times 10^{-4}$. The simplest view of the system of (9a) and (9b)

$$
\begin{aligned}
& \frac{\lambda_{G}}{R_{G} \sqrt{\pi}} \operatorname{erfc}\left(z_{1, G}\right)=\frac{2 \lambda_{C}}{\pi R_{C}} \exp \left(-z_{1, C}\right) ; \\
& \frac{1}{R_{G} \sqrt{\pi}} \frac{\exp \left(-z_{1, G}^{2}\right)}{\operatorname{erfc}\left(z_{1, G}\right)}=\frac{1+z_{1, C}}{R_{C}}
\end{aligned}
$$

corresponds to the range of integration $\left[z_{1, C(G)}, \infty\right)$ and can be used for experimental results from ALICE [25], CMS $[14,15,17,26]$ with exception of the collision energy $\sqrt{s_{N N}}=$ $2.36 \mathrm{TeV}$ [15] in the case of proton-proton collisions, and WA80 [27] for asymmetric nucleus-nucleus collisions $\mathrm{O}+$ $\mathrm{C}, \mathrm{O}+\mathrm{Cu}$. On the other hand, the using of the range of integration $\left[0.0, z_{2, C(G)}\right]$ allows the derivation of the following system from (9a) and (9b):

$$
\begin{aligned}
& \frac{\lambda_{G}}{R_{G} \sqrt{\pi}} \operatorname{erf}\left(z_{2, G}\right)=\frac{2 \lambda_{C}}{\pi R_{C}}\left[1-\exp \left(-z_{2, C}\right)\right] ; \\
& \frac{1}{R_{G} \sqrt{\pi}} \frac{1-\exp \left(-z_{2, G}^{2}\right)}{\operatorname{erf}\left(z_{2, G}\right)} \\
& =\frac{1}{R_{C}} \frac{1-\exp \left(-z_{2, C}\right)\left(1+z_{2, C}\right)}{1-\exp \left(-z_{2, C}\right)} .
\end{aligned}
$$

As expected one can get the ultimate relations (6a) and (6b) from the any systems of ((10a) and (10b)) or ((11a) and (11b)) at $q_{1} \rightarrow 0$ or $q_{2} \rightarrow \infty$, respectively. Therefore system (10a) and (10b) can be replaced by ultimate system of (6a) and (6b) with some accuracy $\varepsilon^{\prime}$ for finite range of $q$ if $q_{1} \leq q_{1}^{h}$ and $q_{2}$ value is large enough to consider this value as $q_{2} \rightarrow \infty$. Similarly, system (11a) and (11b) can be replaced by ultimate system of (6a) and (6b) with some accuracy $\varepsilon^{\prime}$ for finite range of $q$ if $q_{2} \geq q_{2}^{l}$ and $q_{1}$ is small enough to consider it as $q_{1} \rightarrow 0$ for (11a) and (11b). The high/low boundary values $q_{1}^{h} / q_{2}^{l}$ for variables $q_{1} / q_{2}$ are dominated by assigned value of accuracy. For instance, at $\varepsilon^{\prime}=10^{-2}$ the ultimate system of (6a) and (6b) is valid for $q_{1} \lesssim 2 \times 10^{-3} R_{C(G)}$ or $q_{2} \gtrsim 1.3 R_{C(G)}$, that is, $q_{1} \lesssim q_{1}^{h}=2-4 \mathrm{MeV} / \mathrm{c}$ or $q_{2} \gtrsim q_{2}^{l}=1.3-2.6 \mathrm{GeV} / \mathrm{c}$ for proton-proton collisions. The derived estimations are close to the values of $q$ variable used in present experimental analyses of BEC correlations.

These qualitative estimations are confirmed by quantitative analysis below for the $q_{1}$ - and $q_{2}$-dependencies of the Gaussian parameters $\lambda_{G}$ and $R_{G}$ derived for some assigned values of the corresponding BEC parameters for exponential function $\lambda_{C}, R_{C}$ and vice versa.

\subsection{Dependence on $q$ for Desired Cauchy/Gaussian Param-} eters. For Figures 3 and $4 \Omega_{C}$ is considered as a priori known and set of BEC parameters $\left\{\lambda_{G}, R_{G}\right\}$ are studied for Gaussian parametrization (2). Figure 3 shows the $q_{1}$ - and $q_{2}$-dependence of $1 \mathrm{D}$ BEC radius (Figures $3(\mathrm{a})$ and $3(\mathrm{~b})$ ) and strength of correlations (Figures 3(c) and 3(d)) for parametrization (2) with Gaussian function $\Omega_{G}$ at fixed values $\lambda_{C}=\pi / 2$ and $R_{C}=\sqrt{\pi}$. As seen both Gaussian parameters show the similar behavior with changing the integration limits, namely, $\lambda_{G}$ and $R_{G}$ growth with decreasing of the $q_{1,2}$ at fixed another limit of integration. The curves $\lambda_{G}\left(q_{1}\right)$, $R_{G}\left(q_{1}\right)$ approach the asymptotic dashed lines calculated with help of system (10a) and (10b) with increasing of $q_{2}$. The similar situation is observed in Figures 3(b) and 3(d) for curves $R_{G}\left(q_{2}\right), \lambda_{G}\left(q_{2}\right)$ and asymptotic dashed lines calculated with help of system (11a) and (11b) with decreasing of $q_{1}$. As seen the asymptotic lines are achieved at $q_{1} \leqslant 1 \mathrm{MeV} / \mathrm{c}$ (Figures 3(b) and 3(d)) and $q_{2} \gtrsim 0.8 \mathrm{GeV} / \mathrm{c}$ (Figures 3(a) and $3(\mathrm{c})$ ). Furthermore the ultimate values of the Gaussian BEC parameters $\lambda_{G}^{u}$ and $R_{G}^{u}$ are valid with good accuracy for $q_{1}<2.0 \mathrm{MeV} / \mathrm{c}$ and $q_{2}>1.0 \mathrm{GeV} / \mathrm{c}$. The last ranges are in the good agreement with qualitative estimations for protonproton collisions obtained above. It should be emphasized 




(a)

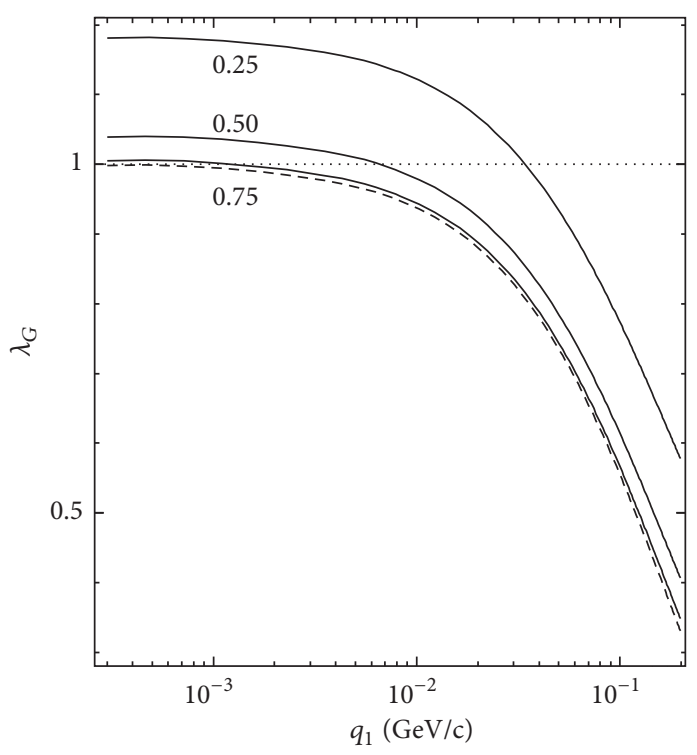

(c)

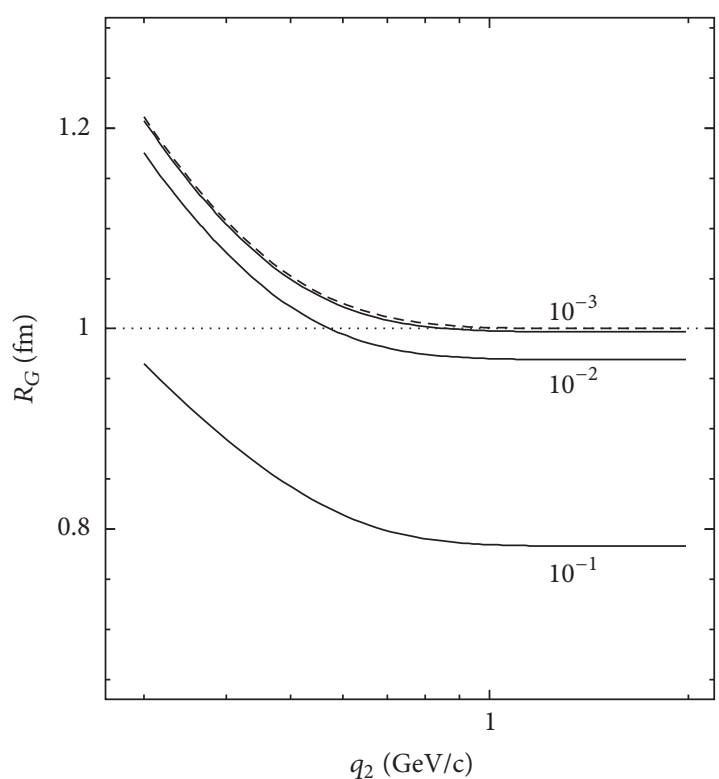

(b)

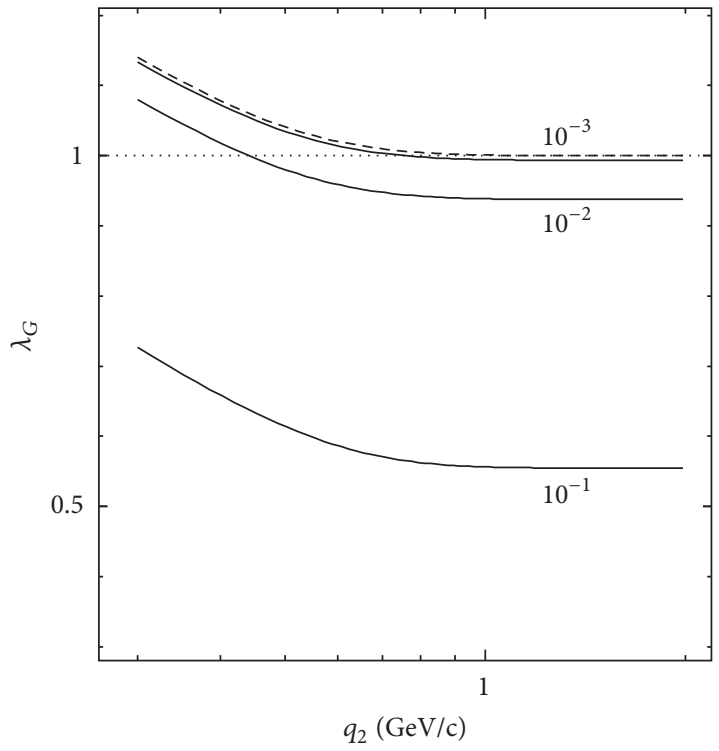

(d)

FIGURE 3: Dependence of 1D BEC radius ( $\mathrm{a}, \mathrm{b})$ and strength of correlations $(\mathrm{c}, \mathrm{d})$ for Gaussian parametrization on low $q_{1}(\mathrm{a}, \mathrm{c})$ and high $q_{2}$ (b, d) limits of integration in the system of (8a) and (8b) for fixed values of the parameters for exponential parametrization: $\lambda_{C}=\pi / 2$ and $R_{C}=\sqrt{\pi} \mathrm{fm}$. The solid lines correspond to the indicated values of the $q_{2}$ for $q_{1}$-dependence (a,c) and to shown values of the $q_{1}$ for $q_{2}$ dependence $(\mathrm{b}, \mathrm{d})$. The dashed lines correspond to the calculations based on system (10a) and (10b) for $q_{1}$-dependence (a, c) and on system (11a) and (11b) for $q_{2}$-dependence (b, d). The thin dotted lines are the ultimate levels $R_{G}=1.0 \mathrm{fm}(\mathrm{a}, \mathrm{b})$ and $\lambda_{G}=1.0$ (c, d) calculated with help of (6a) and (6b) for given values of the set of Cauchy parameters $\left\{\lambda_{C}, R_{C}\right\}$.

that for specific case of exponential $\left(\Omega_{C}\right)$ and Gaussian $\left(\Omega_{G}\right)$ functions the asymptotic $q_{1}$-dependence is achieved for both the $1 \mathrm{D}$ BEC radius (Figure $3(\mathrm{a})$ ) and the strength of correlations (Figure 3(c)) at $q_{2}$ which is much smaller than that for case of two some other centrally symmetric Lévy parameterizations (Figures $3(\mathrm{a})$ and $3(\mathrm{c})$ ). This $q_{2}$ value for case of $\Omega_{C}$ and $\Omega_{G}$ is similar to those used in analyses of experimental CF $\mathrm{C}_{2}(q)$. In Figure 4 the dependencies of relative BEC parameters, namely, $R_{C} / R_{G}(\mathrm{a}, \mathrm{b})$ and $\lambda_{C} / \lambda_{G}(\mathrm{c}, \mathrm{d})$, on $q_{1}(\mathrm{a}, \mathrm{c})$ and $q_{2}(\mathrm{~b}, \mathrm{~d})$ are presented for various assigned values of parameters for Cauchy distribution. The curves are calculated with the simpler system of (10a) and (10b) for $q_{1}$-dependence (Figures 4(a) and 4(c)) and system (11a) and (11b) for $q_{2}$ dependence (Figures 4(b) and 4(d)), respectively. As seen the larger values of Cauchy parameters lead to the larger values of relative BEC parameters. The $q_{1}$-dependence of relative BEC parameters grows faster with increasing of the input values of Cauchy parameters (Figures 4(a) and 4(c)). On the contrary 


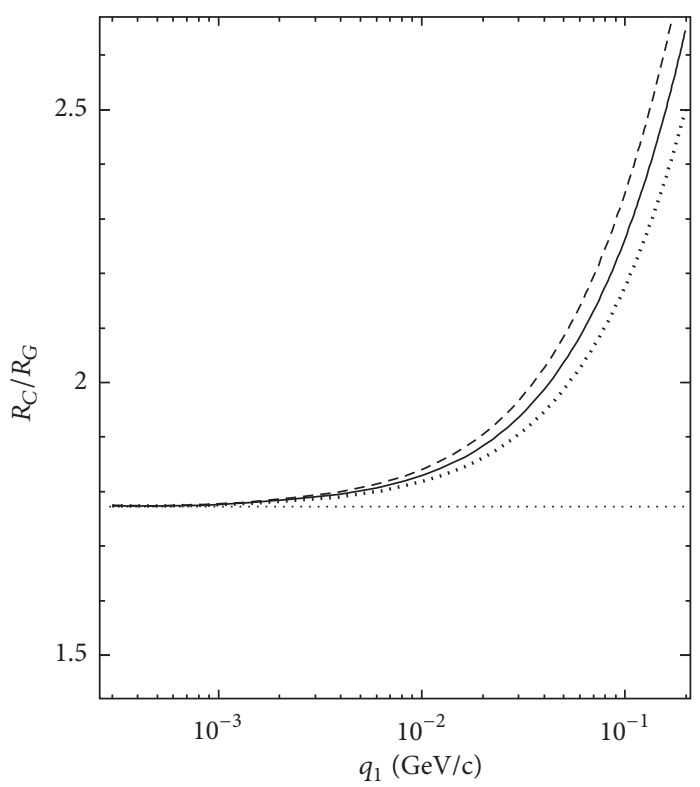

(a)

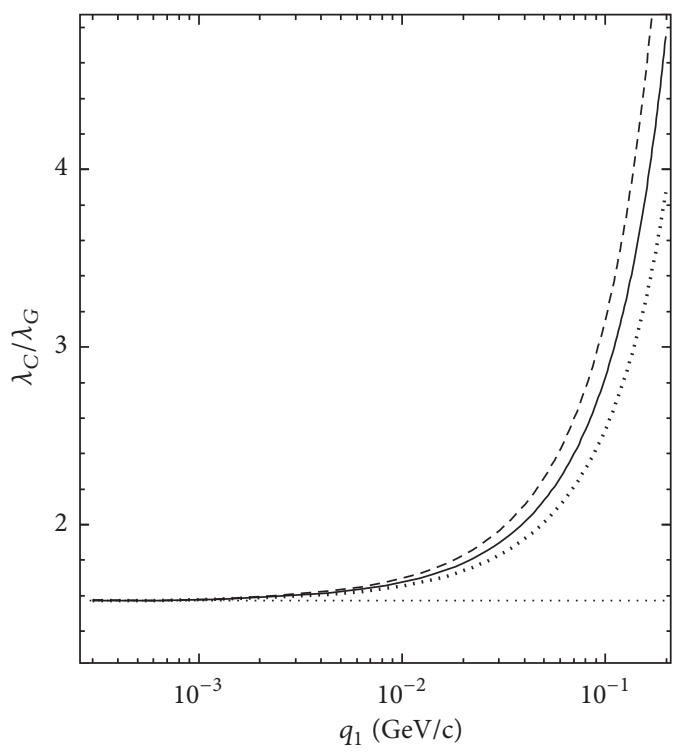

(c)



(b)

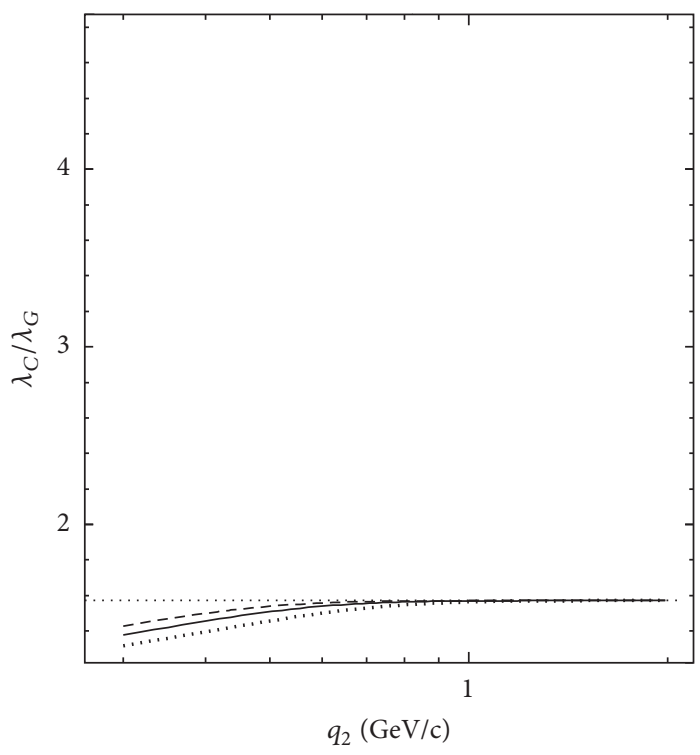

(d)

Figure 4: Dependence of relative 1D BEC radius (a, b) and strength of correlations (c, d) on $q_{1}(\mathrm{a}, \mathrm{c})$ and $q_{2}$ (b, d) for various fixed values of the parameters for exponential parametrization. The calculations are made for simpler range of integration $\left[z_{1, C(G)}, \infty\right)$ with help of system (10a) and (10b) for $q_{1}$-dependence (a, c) and for $\left[0.0, z_{2, C(G)}\right]$ with system (11a) and (11b) for $q_{2}$-dependence (b, d), respectively. The dashed lines correspond to $\lambda_{C}=0.6 \pi, R_{C}=1.2 \sqrt{\pi} \mathrm{fm}$, solid lines $\lambda_{C}=0.5 \pi, R_{C}=\sqrt{\pi} \mathrm{fm}$, and dotted lines $\lambda_{C}=0.4 \pi, R_{C}=0.8 \sqrt{\pi} \mathrm{fm}$. The thin dotted lines are the ultimate levels $R_{C} / R_{G}=\sqrt{\pi}(\mathrm{a}, \mathrm{b})$ and $\lambda_{C} / \lambda_{G}=\pi / 2$ (c, d) corresponding to system (6a) and (6b).

the decrease of the $q_{2}$-dependence of the $R_{C} / R_{G}$ (Figure $4(\mathrm{~b})$ ) and $\lambda_{C} / \lambda_{G}$ (Figure $4(\mathrm{~d})$ ) is slower with increasing of the input values of the $\left\{\lambda_{C}, R_{C}\right\}$. As expected the ultimate levels $R_{C} / R_{G}=\sqrt{\pi}$ (Figures 4(a) and 4(b)) and $\lambda_{C} / \lambda_{G}=\pi / 2$ (Figures $4(\mathrm{c})$ and $4(\mathrm{~d})$ ) shown by thin dotted lines are valid for the same ranges of $q_{1}$ and $q_{2}$ as estimated above for Figure 3.

Figures 5 and 6 show results for opposite direction of calculations; that is, $\Omega_{G}$ is supposed to be a priori known and BEC parameters $\left\{\lambda_{C}, R_{C}\right\}$ for exponential parametrization of $\mathrm{CF} \mathrm{C}_{2}(q)$ are derived. Figure 5 shows the $q_{1}$ - and $q_{2}$ dependence of 1D BEC radius (Figures 5(a) and 5(b)) and strength of correlations (Figures 5(c) and 5(d)) for exponential function $\Omega_{C}$ at fixed values $\lambda_{G}=2 / \pi$ and $R_{G}=1 / \sqrt{\pi}$. The $\forall i=1,2: q_{i}$-dependencies show the opposite behavior for desired parameters of Cauchy source function $R_{C}$ (Figures 5(a) and 5(b)) and $\lambda_{C}$ (Figures 5(c) and 5(d)) with respect to the corresponding dependencies presented in Figure 3 above for another direction of calculation $\left\{\lambda_{C}, R_{C}\right\} \rightarrow\left\{\lambda_{G}, R_{G}\right\}$. These differences are seen in domain of relatively large 


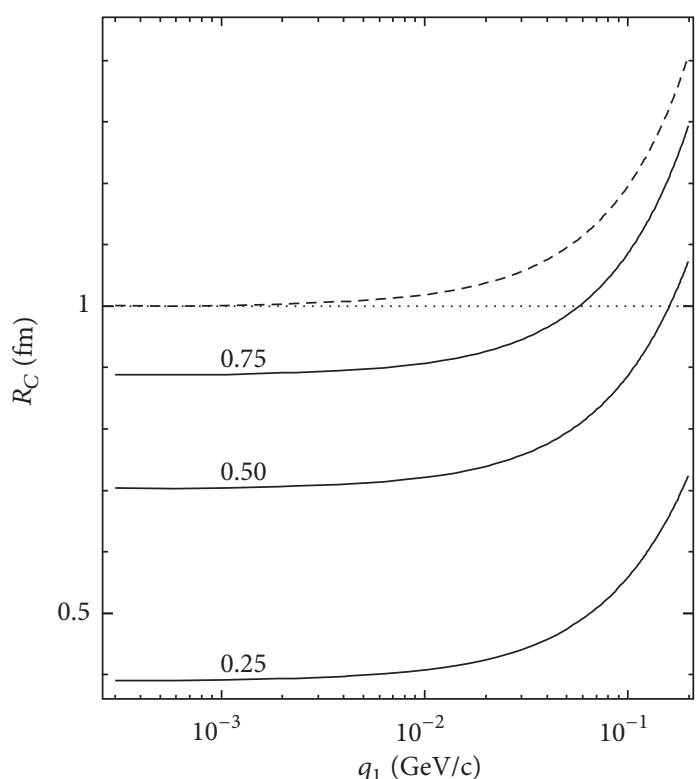

(a)

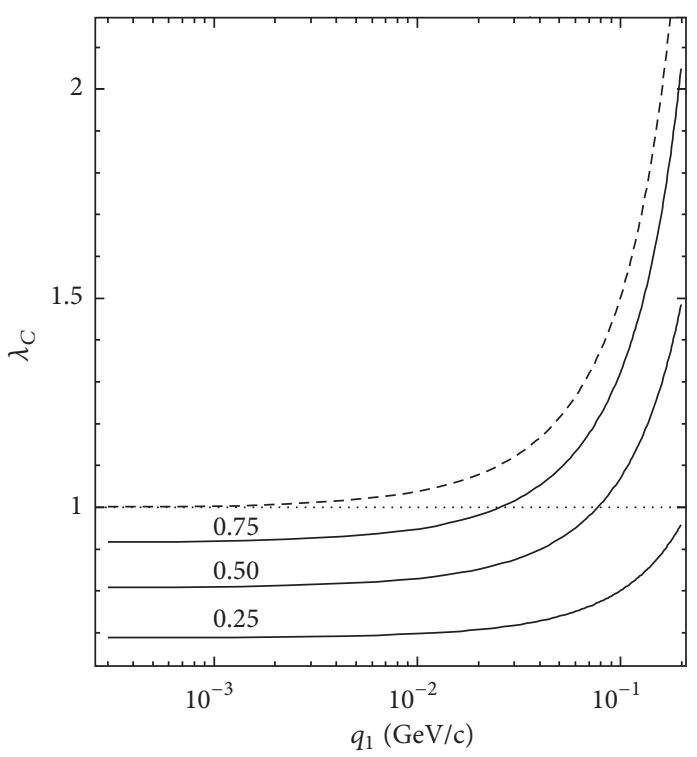

(c)

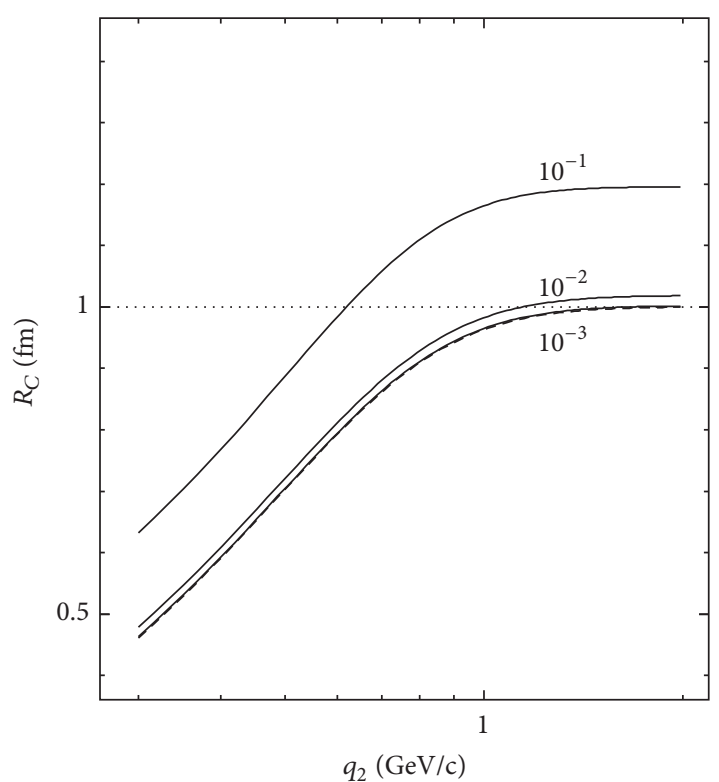

(b)

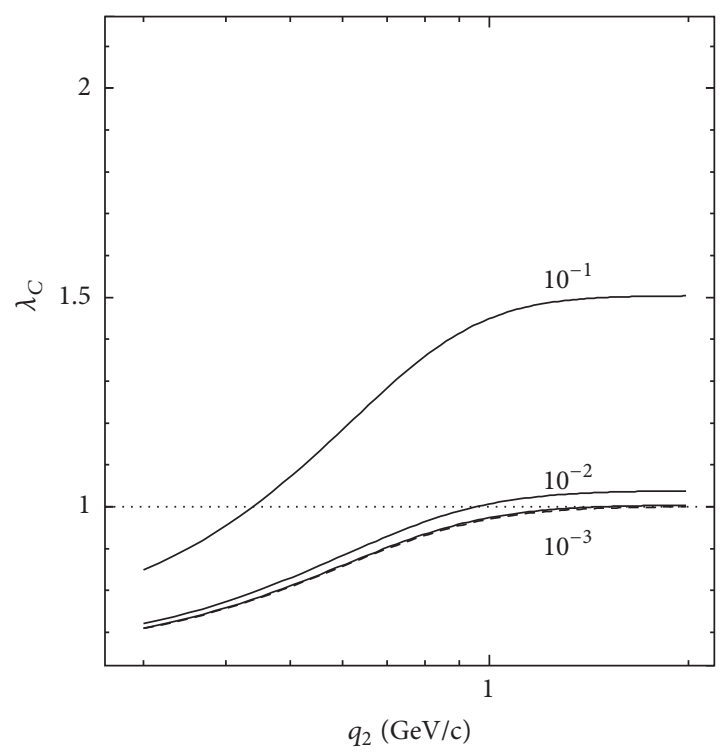

(d)

FIGURE 5: Dependence of 1D BEC radius ( $\mathrm{a}, \mathrm{b}$ ) and strength of correlations $(\mathrm{c}, \mathrm{d})$ for Cauchy distribution on low $q_{1}(\mathrm{a}, \mathrm{c})$ and high $q_{2}$ $(b, d)$ limits of integration in the system of (8a) and (8b) for fixed values of the parameters for Gaussian parametrization: $\lambda_{G}=2 / \pi$ and $R_{G}=1 / \sqrt{\pi} \mathrm{fm}$. The solid lines correspond to the indicated values of the $q_{2}$ for $q_{1}$-dependence $(\mathrm{a}, \mathrm{c})$ and to shown values of the $q_{1}$ for $q_{2}{ }^{-}$ dependence (b, d). The dashed lines correspond to the calculations based on system (10a) and (10b) for $q_{1}$-dependence (a, c) and on system (11a) and (11b) for $q_{2}$-dependence (b, d). The thin dotted lines are the ultimate levels $R_{C}=1.0 \mathrm{fm}(\mathrm{a}, \mathrm{b})$ and $\lambda_{G}=1.0(\mathrm{c}, \mathrm{d})$ calculated with help of (6a) and (6b) for given values of the set of Gaussian parameters $\left\{\lambda_{G}, R_{G}\right\}$.

$q_{1} \gtrsim 10^{-2} \mathrm{GeV} / \mathrm{c}$ for $q_{1}$-dependence and at relatively small $q_{2} \lesssim 0.7 \mathrm{GeV} / \mathrm{c}$ for $q_{2}$-dependence of BEC parameters. Furthermore the $q_{1}$-dependence for parameters from set for $\Omega_{C}$ (Figures 5(a) and 5(c)) approaches the constant at $q_{1} \rightarrow 0$ faster noticeably than that for $R_{G}$ (Figure 3(a)) and $\lambda_{G}$ (Figure 3(c)). The opposite situation is observed for achievement of constants by $q_{2}$-dependence at $q_{2} \rightarrow \infty$. It should be noted that dependencies $R_{C}\left(q_{1}\right)$ and $\lambda_{C}\left(q_{1}\right)$ approach their asymptotic curves calculated with help of system (10a) and (10b) and shown by dashed lines in Figures 5(a) and 5(c) slower than corresponding dependencies for desired Gaussian parameters in Figures 3(a) and 3(c). As a consequence $R_{C}\left(q_{1}\right)$ and $\lambda_{C}\left(q_{1}\right)$ will achieve the asymptotic curves at higher $q_{2}$ than that for Figures 3(a) and 3(c). The asymptotic value of $q_{1} \simeq 10^{-3} \mathrm{GeV} / \mathrm{c}$ is the same for $q_{2}$ dependence for both directions of calculations $\left\{\lambda_{C}, R_{C}\right\} \rightleftarrows$ $\left\{\lambda_{G}, R_{G}\right\}$. Figure 6 demonstrates the dependence of relative BEC parameters, namely, $R_{C} / R_{G}(\mathrm{a}, \mathrm{b})$ and $\lambda_{C} / \lambda_{G}(\mathrm{c}, \mathrm{d})$, 


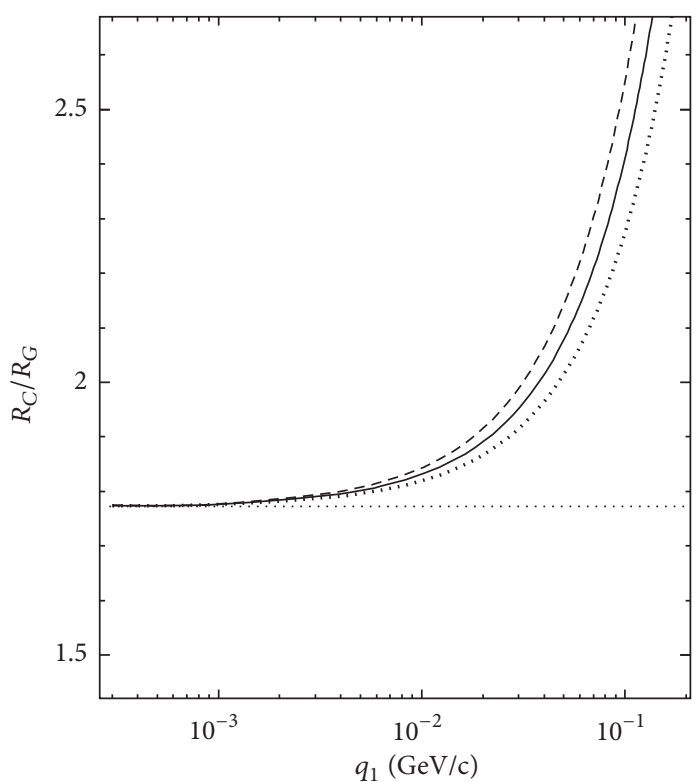

(a)

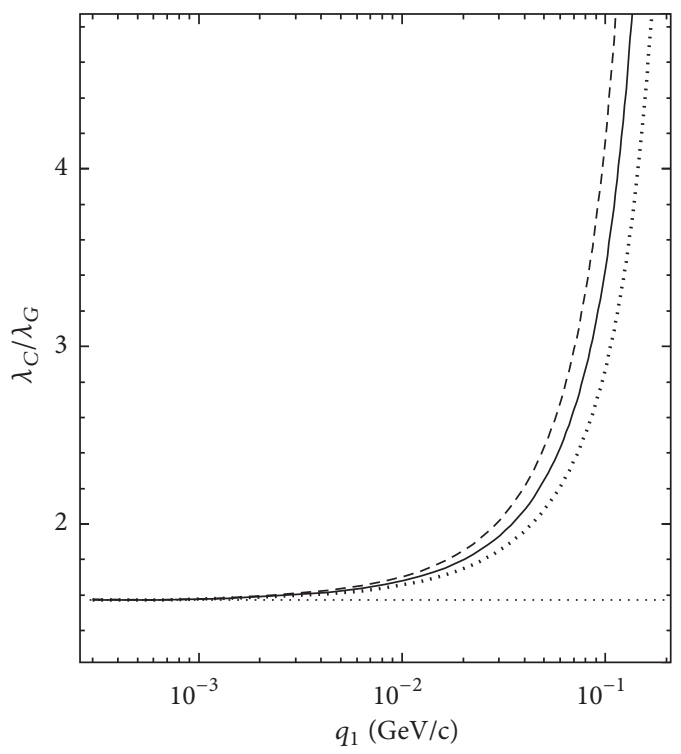

(c)

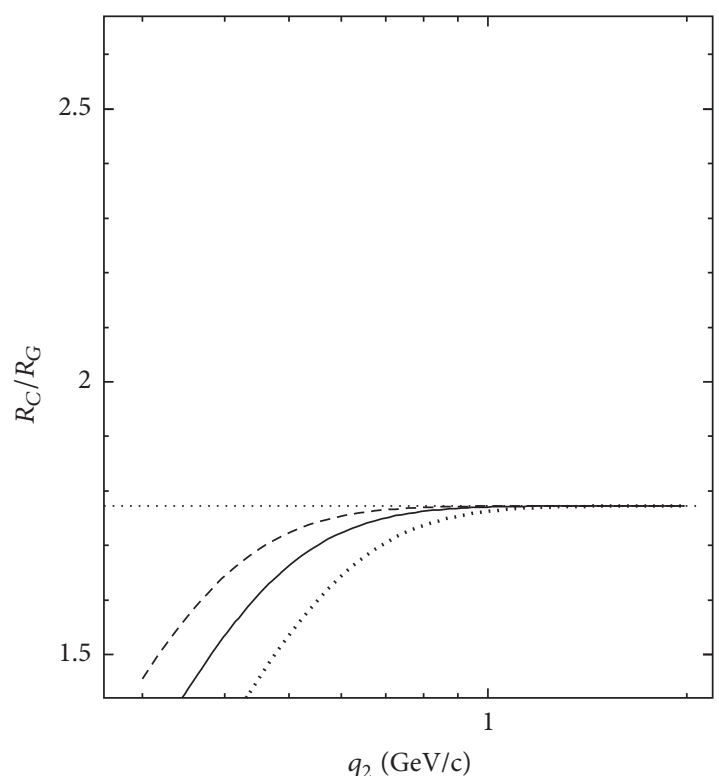

(b)

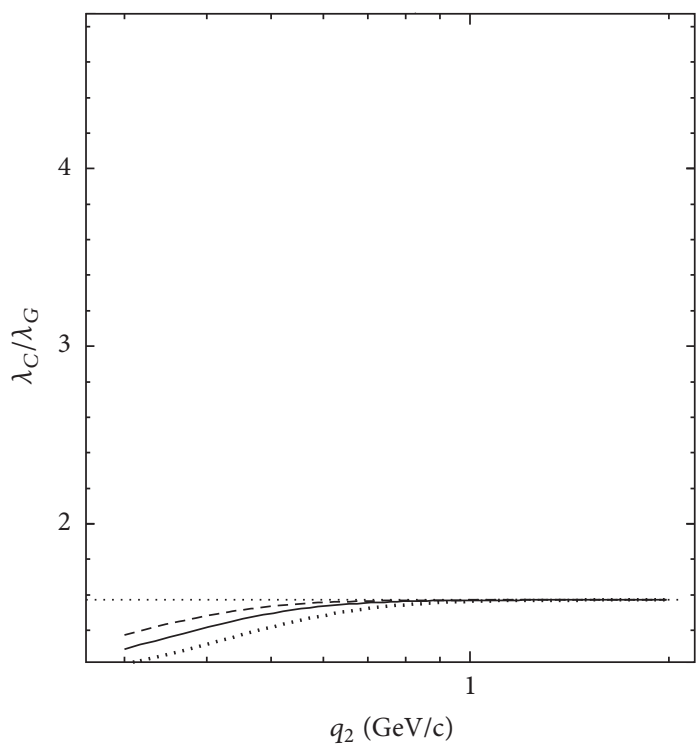

(d)

Figure 6: Dependence of relative 1D BEC radius ( $\mathrm{a}, \mathrm{b}$ ) and strength of correlations $(\mathrm{c}, \mathrm{d})$ on $q_{1}(\mathrm{a}, \mathrm{c})$ and $q_{2}$ (b, d) for various fixed values of the parameters for Gaussian parametrization. The calculations are made for simpler range of integration $\left[z_{1, C(G)}, \infty\right)$ with help of system $(10 \mathrm{a})$ and $(10 \mathrm{~b})$ for $q_{1}$-dependence $(\mathrm{a}, \mathrm{c})$ and for $\left[0.0, z_{2, \mathrm{C}(G)}\right]$ with system (11a) and (11b) for $q_{2}$-dependence (b, d), respectively. The dashed lines correspond to $\lambda_{G}=1.2, R_{G}=1.2 \mathrm{fm}$, solid lines $\lambda_{G}=1.0, R_{G}=1.0 \mathrm{fm}$, and dotted lines $\lambda_{G}=0.8, R_{G}=0.8 \mathrm{fm}$. The thin dotted lines are the ultimate levels $R_{C} / R_{G}=\sqrt{\pi}(\mathrm{a}, \mathrm{b})$ and $\lambda_{C} / \lambda_{G}=\pi / 2(\mathrm{c}, \mathrm{d})$ corresponding to system (6a) and (6b).

on $q_{1}(\mathrm{a}, \mathrm{c})$ and $q_{2}(\mathrm{~b}, \mathrm{~d})$ for various assigned values of parameters for Gaussian parametrization. The simpler system of (10a) and (10b) is used for calculation of $q_{1}$-dependencies in Figures 6(a) and 6(c) and curves on $q_{2}$ (Figures 6(b) and $6(d))$ are derived with help of system (1la) and (11b). In general $\forall i=1,2: q_{i}$-dependencies show similar behavior for corresponding relative $1 \mathrm{D}$ BEC parameters in both cases, Figure 4 and Figure 6 , with some faster changing of $q_{i}{ }^{-}$ dependencies in the second case than that for the first one in domain of relatively large $q_{1} \gtrsim 10^{-2} \mathrm{GeV} / \mathrm{c}$ for $q_{1}$-dependence and at relatively small $q_{2} \leqslant 0.7 \mathrm{GeV} / \mathrm{c}$ for $q_{2}$-dependence of $R_{C} / R_{G}$ and $\lambda_{C} / \lambda_{G}$

Simultaneous consideration of available 1D BEC data analyses for strong interaction processes and Figures 3 and 5 allow the assertion that ultimate relations (6a) and (6b) are not acceptable with reasonable accuracy for most of experimental results with exponential/Gaussian parametrization (2) of $1 \mathrm{D} \mathrm{CF} \mathrm{C}_{2}(q)$. As seen from Figures 4 and 6 even the asymptotic values of relative $1 \mathrm{D}$ BEC parameters $\left\{\lambda_{C} / \lambda_{G}\right.$, $R_{C} / R_{G}$ s can differ up to several times from ultimate values 
calculated with help of the system of (6a) and (6b) in some domains of $q_{1}$ and $q_{2}$ variables. Therefore Figures 3-6 confirm the conclusion formulated above for case of two general view centrally symmetric Lévy parameterizations; namely, for desired 1D BEC parameters the finite values for limits of integrations can lead to the significant difference between values of BEC observables calculated on exact equations and asymptotic/ultimate values calculated on simpler relations.

It should be emphasized that the results of the present paper shown in Figures 3-6 are useful for experimental data analysis as well as for phenomenological studies because it allow, in particular, the quantitative choice between systems of $((6 a)-(11 b))$ for estimations of 1D BEC parameters for specific cases of centrally symmetric Lévy parametrization (2) at $\alpha=1,2$ depending on some features in given experiment.

\section{Comparison with Experimental Results}

Database is created for $1 \mathrm{D}$ BEC results for identical charged pions produced in strong interaction processes in order to verify the mathematical formalisms suggested above. This database is shown in Appendix 5 and it is used as input for calculations below. Experimental results for strength of correlations and 1D source radius are considered for all types of the processes and centrally symmetric Lévy parameterizations (2) and for total available energy range in the paper. The results for most central nucleus-nucleus collisions are usually included in the database because these collisions are used for studying of new features of final state matter [28]. The dependence of 1D BEC parameters on the outgoing charged particle multiplicity, $N_{\text {ch }}$, is widely studied for $p+p$ and $\bar{p}+p$ collisions at least. Therefore the additional separation is made on experimental 1D BEC values deduced for minimum bias and for high multiplicity event classes sometimes (this separation will be stipulated additionally if experimental 1D BEC results are available for various multiplicity event classes in $p+p, \bar{p}+p$ collisions.). This consideration seems important for both the additional verification of mathematics above and the more careful comparison with nucleus-nucleus results. As seen the additional information is required about experimental $q$ ranges for systems ((3a) and (3b)) and ((7a) and $(7 b))$ in comparison with the ultimate relations $((4 a)$ and $(4 b))$ and $((6 a)$ and $(6 b))$. Therefore experimental $q$ ranges are estimated based on the available published data. In Tables 1-4 the statistical errors are shown first and available systematic uncertainties second, unless otherwise specifically indicated; the types of uncertainties (statistical/total, symmetric/asymmetric) are chosen just the same as well as input parameters for the sake of simplicity.

4.1. Relations between Parameters for Cauchy/Gaussian Distribution and Lévy One. The general system of (3a) and (3b) allow us to estimate the $1 \mathrm{D}$ BEC parameters for exponential/Gaussian function $\Omega_{C(G)}$ based on the a priori known parameter values for $\Omega_{L} \equiv \Omega\left(\alpha_{L}, \lambda_{L}, R_{L}\right)$ corresponding to general view of centrally symmetric Lévy distribution and vice versa.
4.1.1. Direction of Calculations $\Omega_{L} \rightarrow \Omega_{C / G}$. The sets $\left\{\lambda_{C}, R_{C}\right\}$ and $\left\{\lambda_{G}, R_{G}\right\}$ are estimated for experimentally known $\Omega_{L}$ [14-16] and finite $q$-ranges with help of (3a) and (3b). The estimations are shown in Table 1 together with the available experimental results and the data for Cauchy distribution are shown on the first line, for Gaussian parametrization, on the second line for certain experiment at given energy. As seen estimations for strength of correlations and $1 \mathrm{D}$ radius calculated with help of (3a) and (3b) agree with experimental values within errors for both the Cauchy and the Gaussian distributions for particle emission points at all energies under study. One can note that estimation for $\lambda_{G}$ coincides with experimental values within total errors only at $\sqrt{s_{N N}}=7 \mathrm{TeV}$. Nevertheless the general system of (3a) and (3b) provides rather well estimations of $1 \mathrm{D}$ BEC parameters for both the Cauchy and the Gaussian distributions. The possibility is considered for application of ultimate relations (4a) and (4b) for the experimental data under study. All estimations from (4a) and (4b) coincide with results from general system (3a) and (3b) within errors with exception of $\lambda_{G}$ for CMS at $\sqrt{s_{N N}}=$ $7 \mathrm{TeV}$. For the last case the estimations from ((3a) and (3b)) and $((4 a)$ and $(4 b))$ coincide with each other within $2 \sigma$. Thus the ultimate relations $(4 \mathrm{a})$ and $(4 \mathrm{~b})$ provide reasonable estimations for 1D BEC parameters in both cases of the Cauchy and the Gaussian distributions within features of modern experiments under consideration, that is, at $q_{1} \sim$ $10^{-2} \mathrm{GeV} / \mathrm{c}, q_{2} \sim 2 \mathrm{GeV} / \mathrm{c}$, and $\alpha_{L} \sim 0.8$ which is close to the region of Lévy index values with weaker changing of $1 D$ BEC parameters (Figure 2).

4.1.2. Direction of Calculations $\Omega_{C / G} \rightarrow \Omega_{L}$. Here the set of $1 \mathrm{D}$ BEC parameters $\left\{\lambda_{L}, R_{L}\right\}$ is estimated at a priori given $\alpha_{L}$ with help of the system of (3a) and (3b) for experimentally known $\Omega_{C}[14,16], \Omega_{G}[15,16]$ and finite $q$-ranges. The estimations for parameters of $\Omega_{L}$ are shown in Table 2 together with the available experimental results and the values of $\lambda_{L}, R_{L}$ derived from experimental analysis with Cauchy distribution are shown on the first line; the second one corresponds to the calculations with data for Gaussian distribution for certain experiment at given energy. The relatively large systematic uncertainties for ATLAS are driven by corresponding error for Lévy index [16]. There is a remarkable agreement between results of calculations and experimental analyses (Table 2): estimations for all 1D BEC parameters coincide with corresponding experimental values within statistical errors with exception of the $\lambda_{L}$ at $\sqrt{s_{N N}}=7 \mathrm{TeV}$ for ATLAS data. For the last case the coincidence between estimations from (3a) and (3b) experiment is achieved within total errors. This conclusion is for both $\Omega_{C} \rightarrow \Omega_{L}$ and $\Omega_{G} \rightarrow \Omega_{L}$ schemes of calculations. Thus the system of general equations (3a) and (3b) provides the high-quality estimations of 1D BEC parameters for general view centrally symmetric Lévy parametrization $\Omega_{L}$ based on the a priori known $\alpha_{L}$ and results for exponential/Gaussian function. One can note the ultimate relations (4a) and (4b) for semi-infinite range of Lorentz invariant quantity $q$ result in reasonable estimations for the set of $1 \mathrm{D}$ BEC parameters $\left\{\lambda_{L}, R_{L}\right\}$ with help of results for exponential function $\Omega_{C}$ as well as for Gaussian one $\Omega_{G}$. 
TABLE 1: Parameter values for exponential and Gaussian parameterizations $\Omega_{C(G)}$.

\begin{tabular}{|c|c|c|c|c|c|c|c|}
\hline \multirow{2}{*}{ Collision } & \multirow{2}{*}{$\begin{array}{l}\sqrt{s_{N N}} \\
\mathrm{GeV}\end{array}$} & \multirow{2}{*}{ Experiment } & \multicolumn{2}{|c|}{ Estimation based on (3a), (3b) } & \multicolumn{2}{|c|}{ Experimental values } & \multirow{2}{*}{ Ref. } \\
\hline & & & $\lambda$ & $R, \mathrm{fm}$ & $\lambda$ & $R, \mathrm{fm}$ & \\
\hline \multirow{6}{*}{$p+p$} & \multirow{2}{*}{900} & \multirow{2}{*}{ CMS } & $0.62 \pm 0.08$ & $1.47 \pm 0.24$ & $0.616 \pm 0.011 \pm 0.029$ & $1.56 \pm 0.02 \pm 0.15$ & [14] \\
\hline & & & $0.35 \pm 0.06$ & $0.81 \pm 0.22$ & $0.32 \pm 0.01$ & $0.98 \pm 0.03$ & [15] \\
\hline & \multirow{4}{*}{7000} & \multirow{2}{*}{ ATLAS } & $0.73 \pm 0.03 \pm 0.54$ & $2.02 \pm 0.11 \pm 1.79$ & $0.701 \pm 0.006 \pm 0.067$ & $2.021 \pm 0.012 \pm 0.281$ & \multirow{2}{*}[16]{} \\
\hline & & & $0.39 \pm 0.01 \pm 0.20$ & $1.07 \pm 0.04 \pm 0.82$ & $0.302 \pm 0.002 \pm 0.019$ & $1.046 \pm 0.005 \pm 0.114$ & \\
\hline & & \multirow{2}{*}{ CMS } & $0.62 \pm 0.06$ & $1.81 \pm 0.23$ & $0.618 \pm 0.009 \pm 0.042$ & $1.89 \pm 0.02 \pm 0.21$ & [14] \\
\hline & & & $0.35 \pm 0.03$ & $0.96 \pm 0.12$ & - & - & - \\
\hline
\end{tabular}

TABLE 2: Parameter values for general Lévy parametrization $\Omega_{L}$ at given $\alpha_{L}$.

\begin{tabular}{|c|c|c|c|c|c|c|c|}
\hline \multirow{2}{*}{ Collision } & \multirow{2}{*}{$\begin{array}{l}\sqrt{s_{N N}} \\
\mathrm{GeV}\end{array}$} & \multirow{2}{*}{ Experiment } & \multicolumn{2}{|c|}{ Estimation based on (3a), (3b) } & \multicolumn{2}{|c|}{ Experimental values } & \multirow{2}{*}{ Ref. } \\
\hline & & & $\lambda$ & $R, \mathrm{fm}$ & $\lambda$ & $R, \mathrm{fm}$ & \\
\hline \multirow{5}{*}{$p+p$} & \multirow{2}{*}{900} & \multirow{2}{*}{ CMS } & $0.85 \pm 0.04 \pm 0.05$ & $2.33 \pm 0.18 \pm 0.23$ & $0.85 \pm 0.06$ & $2.20 \pm 0.17$ & {$[14]$} \\
\hline & & & $0.89 \pm 0.10$ & $3.2 \pm 0.5$ & $0.93 \pm 0.11$ & $2.5 \pm 0.4$ & {$[15]$} \\
\hline & \multirow{3}{*}{7000} & \multirow{2}{*}{ ATLAS } & $0.973 \pm 0.012 \pm 0.332$ & $2.97 \pm 0.06 \pm 1.27$ & $1.02+0.03+0.41$ & $2.96+0.09+1.31$ & {$[16]$} \\
\hline & & & $0.774 \pm 0.010 \pm 0.250$ & $2.86 \pm 0.06 \pm 1.21$ & $1.02 \perp 0.01 \pm 0.41$ & $2.0010 .07 \perp 1.01$ & \\
\hline & & CMS & $0.89 \pm 0.04 \pm 0.07$ & $2.96 \pm 0.17 \pm 0.34$ & $0.90 \pm 0.05$ & $2.83 \pm 0.18$ & {$[14]$} \\
\hline
\end{tabular}

Nevertheless the general system (3a) and (3b) allows the noticeable improvement of the results with respect to (4a) and (4b) for chaoticity $\lambda_{L}$ derived from results for Gaussian distribution. This feature can be expected from Figure 2(d) because curve calculated with (3a) and (3b) differs from the corresponding ultimate $\alpha_{2}$-dependence at values $\alpha_{1} \leqslant 0.8$ which are close to the experimental data (Table 6).

4.2. Relations between Parameters for Cauchy and Gaussian Distributions. The system of (7a) and (7b) derived above is used for estimation of the $1 \mathrm{D}$ BEC parameter values for Gaussian parametrization based on the a priori known values for set $\left\{\lambda_{C}, R_{C}\right\}$ of BEC parameters for exponential parametrization and experimental ranges on $q$ and vice versa. In the subsection the separation is used on various multiplicity event classes in $p+p, \bar{p}+p$ collisions for $1 \mathrm{D}$ BEC results in some experiments. The results for minimum bias events are shown on the first line and for high multiplicity events they are shown on the second line for certain experiment at given energy in Tables 3 and 4.

4.2.1. Direction of Calculations $\Omega_{C} \rightarrow \Omega_{G}$. Parameters for Gaussian function are calculated with help of system (7a) and (7b) and a priori known set $\left\{\lambda_{C}, R_{C}\right\}$. The results are shown in Table 3 together with available published experimental results for the Gaussian set $\left\{\lambda_{G}, R_{G}\right\}$. As seen from Table 3 the estimations for the set of the Gaussian parameters are equal for published results within (total) errors for protonproton collisions with exception of the value of strength of correlations $\lambda_{G}$ in ATLAS minimum bias events at $\sqrt{s_{N N}}=$ $7 \mathrm{TeV}$ and CMS result at collision energy $\sqrt{s_{N N}}=2.36 \mathrm{TeV}$. In the two last cases the agreement is observed within $2 \sigma$. The similar situation is for symmetric nucleus-nucleus collisions; that is, the estimations within the present paper for set of Gaussian parameters $\left\{\lambda_{G}, R_{G}\right\}$ agree with the results of the WA98 experiment [23] within $2 \sigma$. But there is qualitative agreement only between results of calculations with help of (8a) and (8b) and experimental data for $\bar{p}+p$ collisions [24]. Perhaps, this discrepancy is dominated by some features of experiment provided unusually large values of chaoticity for both the exponential and the Gaussian parameterizations of $1 \mathrm{D} \mathrm{CF} \mathrm{C}_{2}(q)$. For asymmetric nuclear interactions the agreement between results of calculations in the present paper and available experimental data is achieved mostly within errors for both $\lambda_{G}$ and the $1 \mathrm{D}$ BEC radius. Only estimations for Gaussian $1 \mathrm{D}$ radius $R_{G}$ in $\mathrm{O}+\mathrm{Ag}$ and for $\lambda_{G}$ in $\mathrm{O}+\mathrm{Au}$ coincide with corresponding results of the WA80 experiment [27] within $2 \sigma$. It should be emphasized that approximate calculations demonstrate the same results as in Table 3 within errors for all consecutive simplifications $((8 \mathrm{a})-(11 \mathrm{~b}))$ which are valid and can be applied for certain experiment. One can note in particular that as expected the ultimate relations (6a) and (6b) work rather well for the CMS results at $\sqrt{s_{N N}}=2.76 \mathrm{TeV}$ with low enough $q_{1} \approx$ $0.6 \mathrm{MeV} / \mathrm{c}$ and high enough $q_{2} \approx 2.0 \mathrm{GeV} / \mathrm{c}$. Thus detailed calculations for case $\Omega_{C} \rightarrow \Omega_{G}$ confirm both the correctness of suggestions made above for certain experiments and the validity of corresponding systems of ((6a)-(11b)).

4.2.2. Direction of Calculations $\Omega_{G} \rightarrow \Omega_{C}$. Values of $1 D$ BEC parameters for exponential function are estimated with help of system (7a) and (7b) and a priori known values for Gaussian BEC quantities $\left\{\lambda_{G}, R_{G}\right\}$. The results are presented in Table 4 together with available published experimental results for the set of BEC parameters $\left\{\lambda_{C}, R_{C}\right\}$ corresponding to the Cauchy distribution function in coordinate space for 
TABLE 3: Parameter values for Gaussian function $\Omega_{G}$ in (2).

\begin{tabular}{|c|c|c|c|c|c|c|c|}
\hline \multirow{2}{*}{ Collision } & \multirow{2}{*}{$\begin{array}{c}\sqrt{\sqrt{s_{N N}}} \\
\mathrm{GeV}\end{array}$} & \multirow{2}{*}{ Experiment } & \multicolumn{2}{|c|}{ Estimation based on (7a), (7b) } & \multicolumn{2}{|c|}{ Experimental values } & \multirow{2}{*}{ Ref. } \\
\hline & & & $\lambda_{G}$ & $R_{G}, \mathrm{fm}$ & $\lambda_{G}$ & $R_{G}, \mathrm{fm}$ & \\
\hline \multirow{11}{*}{$p+p$} & 63.0 & AFS & $0.47 \pm 0.04$ & $0.73 \pm 0.07$ & $0.40 \pm 0.03$ & $0.82 \pm 0.05$ & {$[29]$} \\
\hline & \multirow{4}{*}{900} & \multirow{2}{*}{ ALICE } & $0.305 \pm 0.024$ & $1.00 \pm 0.09_{-0.18}^{+0.06}$ & $0.35 \pm 0.03$ & $1.00 \pm 0.06_{-0.20}^{+0.10}$ & \multirow[b]{2}{*}[25]{} \\
\hline & & & $0.357 \pm 0.025$ & $0.89 \pm 0.06_{-0.18}^{+0.08}$ & $0.310 \pm 0.026$ & $1.18 \pm 0.09_{-0.17}^{+0.07}$ & \\
\hline & & ATLAS & $0.41 \pm 0.01 \pm 0.03$ & $1.00 \pm 0.03 \pm 0.08$ & $0.34 \pm 0.01 \pm 0.03$ & $1.00 \pm 0.03 \pm 0.08$ & {$[30]$} \\
\hline & & \multirow{3}{*}{ CMS } & $0.351 \pm 0.006 \pm 0.013$ & $0.83 \pm 0.01 \pm 0.08$ & $0.32 \pm 0.01$ & $0.98 \pm 0.03$ & \multirow{2}{*}[15]{} \\
\hline & 2360 & & $0.348 \pm 0.030 \pm 0.013$ & $1.04 \pm 0.08 \pm 0.10$ & $0.32 \pm 0.01$ & $0.98 \pm 0.03$ & \\
\hline & 2760 & & $0.366 \pm 0.005 \pm 0.025$ & $0.915 \pm 0.007 \pm 0.116$ & - & - & - \\
\hline & \multirow{4}{*}{7000} & ALICE & $0.719 \pm 0.002 \pm 0.047$ & $1.148 \pm 0.007_{-0.02}^{+0.04}$ & $0.645 \pm 0.003 \pm 0.047$ & $1.430 \pm 0.005_{-0.30}^{+0.16}$ & {$[31]$} \\
\hline & & \multirow{2}{*}{ ATLAS } & $0.381 \pm 0.003 \pm 0.022$ & $1.092 \pm 0.005 \pm 0.074$ & $0.327 \pm 0.002 \pm 0.020$ & $1.130 \pm 0.005 \pm 0.086$ & \multirow{2}{*}[30]{} \\
\hline & & & $0.266 \pm 0.009 \pm 0.015$ & $1.25 \pm 0.03 \pm 0.09$ & $0.251 \pm 0.010 \pm 0.018$ & $1.38 \pm 0.04 \pm 0.12$ & \\
\hline & & CMS & $0.344 \pm 0.005 \pm 0.018$ & $1.00 \pm 0.01 \pm 0.10$ & - & - & - \\
\hline $\bar{p}+p$ & 1.89 & CPLEAR & $2.332 \pm 0.025$ & $0.972 \pm 0.014$ & $1.96 \pm 0.03$ & $1.04 \pm 0.01$ & {$[24]$} \\
\hline$p+\mathrm{Pb}$ & 5020 & CMS & $0.358 \pm 0.007 \pm 0.021$ & $1.70 \pm 0.02 \pm 0.12$ & - & - & - \\
\hline $\mathrm{Pb}+\mathrm{Pb}$ & 17.3 & WA98 & $0.327 \pm 0.008$ & $6.51 \pm 0.10$ & $0.307 \pm 0.008$ & $6.83 \pm 0.10$ & [23] \\
\hline $\mathrm{O}+\mathrm{C}$ & \multirow{4}{*}{19.4} & \multirow{4}{*}{ WA80 } & $0.44 \pm 0.05$ & $2.8 \pm 0.3$ & $0.40 \pm 0.03$ & $2.90 \pm 0.21$ & \multirow{4}{*}[27]{} \\
\hline $\mathrm{O}+\mathrm{Cu}$ & & & $0.24 \pm 0.07$ & $2.53 \pm 0.11$ & $0.17 \pm 0.03$ & $2.35 \pm 0.11$ & \\
\hline $\mathrm{O}+\mathrm{Ag}$ & & & $0.28 \pm 0.10$ & $2.71 \pm 0.11$ & $0.17 \pm 0.04$ & $2.44 \pm 0.11$ & \\
\hline $\mathrm{O}+\mathrm{Au}$ & & & $0.110 \pm 0.015$ & $1.63 \pm 0.05$ & $0.085 \pm 0.007$ & $1.68 \pm 0.06$ & \\
\hline
\end{tabular}

TABLE 4: Parameter values for exponential function $\Omega_{C}$ in (2).

\begin{tabular}{|c|c|c|c|c|c|c|c|}
\hline \multirow{2}{*}{ Collision } & \multirow{2}{*}{$\begin{array}{l}\sqrt{s_{N N}}, \\
\mathrm{GeV}\end{array}$} & \multirow{2}{*}{ Experiment } & \multicolumn{2}{|c|}{ Estimation based on (7a), (7b) } & \multicolumn{2}{|c|}{ Experimental values } & \multirow{2}{*}{ Ref. } \\
\hline & & & $\lambda_{\mathrm{C}}$ & $R_{C}, \mathrm{fm}$ & $\lambda_{\mathrm{C}}$ & $R_{C}, \mathrm{fm}$ & \\
\hline \multirow{12}{*}{$p+p$} & 7.21 & E766 & $1.63 \pm 0.10$ & $2.29 \pm 0.08$ & - & - & - \\
\hline & 26.0 & NA23 & $1.4 \pm 0.7$ & $2.6 \pm 0.7$ & - & - & - \\
\hline & 63.0 & AFS & $0.67 \pm 0.05$ & $1.50 \pm 0.10$ & $0.77 \pm 0.07$ & $1.32 \pm 0.13$ & [29] \\
\hline & 200 & STAR & $0.588 \pm 0.010$ & $2.43 \pm 0.04 \pm 0.26$ & - & - & - \\
\hline & \multirow{3}{*}{900} & \multirow{2}{*}{ ALICE } & $0.63 \pm 0.06$ & $1.89 \pm 0.12_{-0.40}^{+0.20}$ & $0.63 \pm 0.05$ & $1.67 \pm 0.12_{-0.35}^{+0.16}$ & \multirow{2}{*}{ [25] } \\
\hline & & & $0.57 \pm 0.06$ & $2.26 \pm 0.19_{-0.34}^{+0.40}$ & $0.55 \pm 0.05$ & $1.90 \pm 0.18_{-0.36}^{+0.11}$ & \\
\hline & & ATLAS & $0.62 \pm 0.03 \pm 0.08$ & $1.84 \pm 0.07 \pm 0.20$ & $0.74 \pm 0.03 \pm 0.09$ & $1.83 \pm 0.07 \pm 0.20$ & {$[30]$} \\
\hline & & \multirow{2}{*}{ CMS } & $0.57 \pm 0.02$ & $1.85 \pm 0.06$ & $0.616 \pm 0.011 \pm 0.029$ & $1.56 \pm 0.02 \pm 0.15$ & {$[14,15]$} \\
\hline & 2360 & & $0.60 \pm 0.03$ & $1.86 \pm 0.07$ & $0.66 \pm 0.07 \pm 0.05$ & $1.99 \pm 0.18 \pm 0.24$ & {$[15]$} \\
\hline & \multirow{3}{*}{7000} & ALICE & $1.104 \pm 0.006 \pm 0.112$ & $2.627 \pm 0.010_{-0.626}^{+0.320}$ & $1.180 \pm 0.005 \pm 0.084$ & $2.038 \pm 0.014_{-0.046}^{+0.083}$ & [31] \\
\hline & & \multirow{2}{*}{ ATLAS } & $0.627 \pm 0.005 \pm 0.058$ & $2.163 \pm 0.012 \pm 0.209$ & $0.718 \pm 0.006 \pm 0.062$ & $2.067 \pm 0.012 \pm 0.182$ & \multirow{2}{*}{ [30] } \\
\hline & & & $0.521 \pm 0.027 \pm 0.055$ & $2.76 \pm 0.10 \pm 0.29$ & $0.531 \pm 0.024 \pm 0.046$ & $2.46 \pm 0.08 \pm 0.22$ & \\
\hline $\bar{p}+p$ & 1.89 & CPLEAR & $4.21 \pm 0.09$ & $2.079 \pm 0.028$ & $4.79 \pm 0.10$ & $1.89 \pm 0.04$ & {$[24]$} \\
\hline $\mathrm{Au}+\mathrm{Au}$ & 4.86 & E802 & $0.86 \pm 0.08$ & $12.4 \pm 0.6$ & - & - & - \\
\hline \multirow{2}{*}{$\mathrm{Pb}+\mathrm{Pb}$} & \multirow{2}{*}{17.3} & NA44 & $1.06 \pm 0.10$ & $15.1 \pm 0.9$ & - & - & - \\
\hline & & WA98 & $0.69 \pm 0.02$ & $14.1 \pm 0.3$ & $0.718 \pm 0.023$ & $13.34 \pm 0.26$ & {$[23]$} \\
\hline $\mathrm{Au}+\mathrm{Au}$ & 130 & STAR & $0.99 \pm 0.03 \pm 0.08$ & $13.0 \pm 0.3 \pm 0.9$ & - & - & - \\
\hline $\mathrm{Si}+\mathrm{Al}$ & \multirow{2}{*}{5.41} & \multirow{2}{*}{ E802 } & $1.23 \pm 0.08$ & $8.4 \pm 0.3$ & - & - & - \\
\hline $\mathrm{Si}+\mathrm{Au}$ & & & $0.95 \pm 0.05$ & $9.4 \pm 0.3$ & - & - & - \\
\hline $\mathrm{S}+\mathrm{Pb}$ & 17.3 & NA44 & $0.81 \pm 0.05$ & $7.8 \pm 0.6$ & - & - & - \\
\hline $\mathrm{O}+\mathrm{C}$ & \multirow{4}{*}{19.4} & \multirow{4}{*}{ WA80 } & $0.85 \pm 0.08$ & $5.9 \pm 0.5$ & $0.92 \pm 0.13$ & $5.7 \pm 0.7$ & \multirow{4}{*}[27]{} \\
\hline $\mathrm{O}+\mathrm{Cu}$ & & & $0.34 \pm 0.06$ & $4.7 \pm 0.2$ & $0.49 \pm 0.14$ & $5.05 \pm 0.25$ & \\
\hline $\mathrm{O}+\mathrm{Ag}$ & & & $0.34 \pm 0.09$ & $4.9 \pm 0.2$ & $0.59 \pm 0.21$ & $5.46 \pm 0.24$ & \\
\hline $\mathrm{O}+\mathrm{Au}$ & & & $0.156 \pm 0.014$ & $3.19 \pm 0.13$ & $0.20 \pm 0.03$ & $3.07 \pm 0.12$ & \\
\hline
\end{tabular}


particle emission points. For $p+p$ collisions there is agreement between estimations for parameters for exponential parametrization of $\mathrm{CF}_{2}(q)$ calculated with (7a) and (7b) and experimental results within (total) errors with exception of the $1 \mathrm{D} \mathrm{BEC}$ radius $R_{\mathrm{C}}$ in CMS at $\sqrt{s_{N N}}=0.9 \mathrm{TeV}$. In the last case results from calculation and experiment coincide within $2 \sigma$. The similar situation is observed for nucleus-nucleus collisions: estimations for parameters of exponential function $\Omega_{C}$ derived with (7a) and (7b) agree with corresponding experimental results mostly within $1 \sigma$, but the coincidence is achieved within $2 \sigma$ for $R_{\mathrm{C}}$ in both the $\mathrm{Pb}+\mathrm{Pb}$ collisions at $\sqrt{s_{N N}}=17.3 \mathrm{GeV}[23]$ and the $\mathrm{O}+\mathrm{Ag}$ reactions at $\sqrt{s_{N N}}=$ $19.4 \mathrm{GeV}$ [27]. The estimations of $1 \mathrm{D}$ BEC parameters $\lambda_{C}$, $R_{C}$ obtained for $\bar{p}+p$ with help of (8a) and (8b) are in qualitative agreement with corresponding experimental results [24] even for the case of unusually large chaoticity. Thus the system of (7a) and (7b) provides quite reasonable estimations for parameters for exponential function based on the a priori known values of $1 \mathrm{D}$ BEC observables for Gaussian function in various strong interaction processes at all available experimental energies.

In summary of the section, the systems of ((3a) and (3b)) and $((7 a)$ and $(7 b))$ provide correct estimations for both desired BEC parameters, namely, the strength of correlations and the $1 \mathrm{D}$ radius in the case of centrally symmetric Lévy distribution $\Omega_{L}$ as well as for specific Cauchy and Gaussian ones. In general the estimations show remarkable agreement with available experimental data. Thus the systems of equations suggested in Sections 2 and 3 can be useful in experimental data analysis as well as in phenomenological study for estimation of unknown values of BEC parameters for some parametrization (2) of $1 \mathrm{D} \mathrm{CF} \mathrm{C}_{2}(q)$ based on the available values of $\lambda$ and $R$ for another centrally symmetric Lévy distribution. As seen from Tables 1-4 the new estimations are obtained for 1D BEC parameters in $\Omega_{C}, \Omega_{G}$ in many cases for which the corresponding experimental results are absent. Thus the systems of equations derived within the framework of this paper allow the expansion of the available ensemble of values for $1 \mathrm{D}$ BEC parameters $\lambda$ and $R$ which it is useful for future investigations.

\section{Summary}

The case is investigated for smooth approximation of the one experimental 1D Bose-Einstein correlation function by two various centrally symmetric Lévy parameterizations. It is suggested that lowest moments of corresponding distributions are equal approximately. Then the relations are derived between sets of $1 \mathrm{D}$ BEC observables, namely, strength of correlations and source radius, for two general view centrally symmetric Lévy parameterizations under consideration for the first time. The relations obtained in the paper take into account the finiteness of range of Lorentz invariant fourmomentum difference in experimental studies. Detailed analysis results in the systems of transcendental equations for various finite ranges of the Lorentz invariant four-momentum difference in the specific case of the exponential and Gaussian parameterizations for correlation function. It is shown that finite range of $q$ should be taken into account and corresponding systems of equations should be used for derivation of set $\{\lambda, R\}$ based on the a priori known values of corresponding parameters for both cases of the two general view centrally symmetric Lévy parameterizations and the two specific functions (exponential and Gaussian) most used in experimental studies. The ultimate relations derived for semiinfinite range of $q$ can be utilized carefully for experimental analysis and these equations can produce the reasonable estimations for 1D BEC parameters for ranges of Lévy indexes $\forall i=1,2: \alpha_{i} \gtrsim 1$ only. Furthermore it is demonstrated that the corresponding ultimate relations for specific case of Cauchy and Gaussian distributions for source in coordinate space produce the reasonable estimations of $1 \mathrm{D}$ BEC parameters for few modern experimental analyses only. The mathematical formalism suggested within the framework of the preset paper is verified with help of experimental results obtained for wide set of strong interaction processes in all available energy range. The two pairs of distributions are considered: general view centrally symmetric Lévy one with specific case (Cauchy/Gaussian); two specific Cauchy and Gaussian distributions. For both cases verifications are made for both directions of calculations. Namely, the calculations have been made for estimation of 1D BEC observables for Cauchy/ Gaussian function based on the a priori known values of parameters of general Lévy parametrization and vice versa, for estimations of Gaussian parameters based on the a priori known values of observables for Cauchy distribution and vice versa. Comparison shows the quantitative agreement between estimations derived with help of mathematical formalism developed in the paper and most of available experimental results for both pairs consisting of the general view centrally symmetric Lévy parametrization and specific (exponential/Gaussian) function and the two specific source distributions (Cauchy and Gaussian) most used in experimental studies for any direction of calculations.

\section{Appendix}

\section{Data for 1D BEC Parameters in Strong Interactions}

In this Appendix experimental database is shown in Tables 5 and 6 for $1 \mathrm{D}$ BEC parameters for identical charged pions produced in $p+p, \bar{p}+p$, and $A_{1}+A_{2}$ interactions (in Table 5 total uncertainties are shown for exponential parametrization in CPLEAR [24] and for NA44 experiment [42]). Some of the numerical values are used in Section 4 . The pion pairs with low average transverse momentum, $\left\langle k_{T}\right\rangle$, are considered for all types of strong interaction processes. The results for most central nucleus-nucleus collisions are used and as a consequence additional separation is made for $p+p$ collisions on minimum bias and high multiplicity events if corresponding experimental 1D BEC values are available. In the last case the results for minimum bias events are shown on the first line, for high multiplicity events, and on the second line, for certain experiment at given energy. 
TABLE 5: Experimental results for special cases of parametrization (2).

\begin{tabular}{|c|c|c|c|c|c|c|c|}
\hline \multirow{2}{*}{ Collision } & \multirow{2}{*}{$\begin{array}{l}\sqrt{s_{N N}} \\
\mathrm{GeV}\end{array}$} & \multirow{2}{*}{ Experiment } & \multicolumn{2}{|c|}{ Exponential function $\Omega_{C}$} & \multicolumn{2}{|c|}{ Gaussian function $\Omega_{G}$} & \multirow{2}{*}{ Ref. } \\
\hline & & & $\lambda_{C}$ & $R_{\mathrm{C}}, \mathrm{fm}$ & $\lambda_{G}$ & $R_{G}, \mathrm{fm}$ & \\
\hline \multirow{18}{*}{$p+p$} & 7.21 & E766 & - & - & $0.466 \pm 0.015$ & $0.95 \pm 0.03$ & [32] \\
\hline & 26.0 & NA23 & - & - & $0.32 \pm 0.08$ & $1.02 \pm 0.20$ & {$[33]$} \\
\hline & 27.4 & NA27 & - & - & - & $1.20 \pm 0.03$ & {$[34]$} \\
\hline & 31.0 & & - & - & $0.35 \pm 0.04$ & $1.01 \pm 0.08$ & \\
\hline & 44.0 & ABCDHW & - & - & $0.42 \pm 0.04$ & $1.13 \pm 0.07$ & {$[35]$} \\
\hline & 62.0 & & - & - & $0.42 \pm 0.08$ & $1.69 \pm 0.25$ & \\
\hline & 63.0 & AFS & $0.77 \pm 0.07$ & $1.32 \pm 0.13$ & $0.40 \pm 0.03$ & $0.82 \pm 0.05$ & [29] \\
\hline & 200 & STAR & - & - & $0.345 \pm 0.005$ & $1.32 \pm 0.02 \pm 0.13$ & {$[36]$} \\
\hline & \multirow{4}{*}{900} & \multirow{2}{*}{ ALICE } & $0.63 \pm 0.05$ & $1.87 \pm 0.12_{-0.35}^{+0.16}$ & $0.35 \pm 0.03$ & $1.00 \pm 0.06_{-0.20}^{+0.10}$ & \multirow{2}{*}[25]{} \\
\hline & & & $0.55 \pm 0.05$ & $1.90 \pm 0.18_{-0.36}^{+0.11}$ & $0.310 \pm 0.026$ & $1.184 \pm 0.092_{-0.168}^{+0.067}$ & \\
\hline & & ATLAS & $0.74 \pm 0.03 \pm 0.09$ & $1.83 \pm 0.07 \pm 0.20$ & $0.34 \pm 0.01 \pm 0.03$ & $1.00 \pm 0.03 \pm 0.08$ & {$[30]$} \\
\hline & & & $0.616 \pm 0.011 \pm 0.029$ & $1.56 \pm 0.02 \pm 0.15$ & $0.32 \pm 0.01$ & $0.98 \pm 0.03$ & {$[14,15]$} \\
\hline & 2360 & CMS & $0.66 \pm 0.07 \pm 0.05$ & $1.99 \pm 0.18 \pm 0.24$ & $0.32 \pm 0.01$ & $0.98 \pm 0.03$ & {$[15]$} \\
\hline & 2760 & & $0.808 \pm 0.017 \pm 0.062$ & $2.35 \pm 0.07 \pm 0.31$ & - & - & [17] \\
\hline & \multirow{4}{*}{7000} & ALICE & $1.180 \pm 0.005 \pm 0.084$ & $2.038 \pm 0.014_{-0.046}^{+0.083}$ & $0.645 \pm 0.003 \pm 0.047$ & $1.430 \pm 0.005_{-0.300}^{+0.158}$ & {$[31]$} \\
\hline & & ATIAS & $0.718 \pm 0.006 \pm 0.062$ & $2.067 \pm 0.012 \pm 0.182$ & $0.327 \pm 0.002 \pm 0.020$ & $1.130 \pm 0.005 \pm 0.086$ & \multirow{2}{*}{ [30] } \\
\hline & & & $0.531 \pm 0.024 \pm 0.046$ & $2.46 \pm 0.08 \pm 0.22$ & $0.251 \pm 0.010 \pm 0.018$ & $1.38 \pm 0.04 \pm 0.12$ & \\
\hline & & CMS & $0.618 \pm 0.009 \pm 0.042$ & $1.89 \pm 0.02 \pm 0.21$ & - & - & [14] \\
\hline \multirow{3}{*}{$\bar{p}+p$} & 1.89 & CPLEAR & $4.79 \pm 10$ & $1.89 \pm 0.04$ & $1.96 \pm 0.03$ & $1.04 \pm 0.01$ & {$[24]$} \\
\hline & 1800 & E735 & - & - & $0.24 \pm 0.02$ & $1.46 \pm 0.10 \pm 0.23$ & [37] \\
\hline & 1960 & $\mathrm{CDF}$ & $0.89 \pm 0.03$ & $1.67 \pm 0.05$ & $0.50 \pm 0.04$ & $1.79 \pm 0.08$ & {$[38]$} \\
\hline \multirow{3}{*}{$p+\mathrm{Pb}$} & \multirow{3}{*}{5020} & ALICE & $1.230 \pm 0.016_{-0.141}^{+0.088}$ & $4.82 \pm 0.05_{-0.72}^{+0.25}$ & $0.603 \pm 0.006 \pm 0.056$ & $2.780 \pm 0.018_{-0.668}^{+0.418}$ & {$[31]$} \\
\hline & & ATLAS & - & $5.32 \pm 0.06_{-0.14}^{+0.50}$ & - & - & [39] \\
\hline & & CMS & $0.81 \pm 0.02 \pm 0.07$ & $3.55 \pm 0.05 \pm 0.30$ & - & - & {$[26]$} \\
\hline $\mathrm{Au}+\mathrm{Au}$ & 4.86 & E802 & - & - & $0.44 \pm 0.03$ & $6.32 \pm 0.29$ & {$[40]$} \\
\hline $\mathrm{Au}+\mathrm{Pb}$ & \multirow{3}{*}{17.3} & NA49 & - & - & $0.560 \pm 0.023$ & - & {$[41]$} \\
\hline \multirow{2}{*}{$\mathrm{Pb}+\mathrm{Pb}$} & & NA44 & - & - & $0.52 \pm 0.04$ & $7.6 \pm 0.4$ & {$[42]$} \\
\hline & & WA98 & $0.718 \pm 0.023$ & $13.34 \pm 0.26$ & $0.307 \pm 0.008$ & $6.83 \pm 0.10$ & {$[23]$} \\
\hline \multirow{2}{*}{$\mathrm{Au}+\mathrm{Au}$} & \multirow{2}{*}{130} & PHENIX & - & - & - & $6.0 \pm 0.3$ & {$[43]$} \\
\hline & & STAR & - & - & $0.450 \pm 0.009 \pm 0.027$ & $6.30 \pm 0.12 \pm 0.38$ & {$[18]$} \\
\hline $\mathrm{Pb}+\mathrm{Pb}$ & 2760 & ALICE & $1.830 \pm 0.003 \pm 0.156$ & $19.85 \pm 0.02 \pm 0.28$ & $0.689 \pm 0.001 \pm 0.096$ & $9.70 \pm 0.06 \pm 1.17$ & {$[31]$} \\
\hline $\mathrm{Si}+\mathrm{Al}$ & \multirow{2}{*}{5.41} & \multirow{2}{*}{ E802 } & - & - & $0.68 \pm 0.04$ & $4.42 \pm 0.16$ & \multirow{2}{*}[40]{} \\
\hline $\mathrm{Si}+\mathrm{Au}$ & & & - & - & $0.511 \pm 0.026$ & $4.91 \pm 0.15$ & \\
\hline $\mathrm{S}+\mathrm{Pb}$ & 17.3 & NA44 & - & - & $0.42 \pm 0.02$ & $4.00 \pm 0.27$ & {$[42]$} \\
\hline $\mathrm{O}+\mathrm{C}$ & \multirow{5}{*}{19.4} & \multirow{3}{*}{ WA80 } & $0.92 \pm 0.13$ & $5.7 \pm 0.7$ & $0.40 \pm 0.03$ & $2.90 \pm 0.21$ & \multirow{4}{*}{ [27] } \\
\hline $\mathrm{O}+\mathrm{Cu}$ & & & $0.49 \pm 0.14$ & $5.05 \pm 0.25$ & $0.17 \pm 0.03$ & $2.35 \pm 0.11$ & \\
\hline $\mathrm{O}+\mathrm{Ag}$ & & & $0.59 \pm 0.21$ & $5.46 \pm 0.24$ & $0.17 \pm 0.04$ & $2.44 \pm 0.11$ & \\
\hline $\mathrm{O}+\mathrm{A} u$ & & & $0.20 \pm 0.03$ & $3.07 \pm 0.12$ & $0.085 \pm 0.007$ & $1.68 \pm 0.06$ & \\
\hline $\mathrm{U}+\mathrm{Au}$ & & NA35 & - & - & $0.29 \pm 0.03$ & $4.00 \pm 0.20$ & {$[44]$} \\
\hline
\end{tabular}

TABLE 6: Experimental results for general centrally symmetric Lévy parametrization (2).

\begin{tabular}{|c|c|c|c|c|c|c|}
\hline \multirow{2}{*}{ Collision } & \multirow{2}{*}{$\sqrt{s_{N N}}, \mathrm{GeV}$} & \multirow{2}{*}{ Experiment } & \multicolumn{3}{|c|}{ General view function $\Omega_{L}$} & \multirow{2}{*}{ Ref. } \\
\hline & & & $\lambda_{L}$ & $R_{L}, \mathrm{fm}$ & $\alpha_{L}$ & \\
\hline \multirow{4}{*}{$p+p$} & \multirow{2}{*}{900} & \multirow{2}{*}{ CMS } & $0.93 \pm 0.11$ & $2.5 \pm 0.4$ & $0.76 \pm 0.06$ & {$[15]$} \\
\hline & & & $0.85 \pm 0.06$ & $2.20 \pm 0.17$ & $0.81 \pm 0.03$ & {$[14]$} \\
\hline & \multirow{2}{*}{7000} & ATLAS & $1.02 \pm 0.03 \pm 0.41$ & $2.96 \pm 0.09 \pm 1.31$ & $0.81 \pm 0.01 \pm 0.18$ & {$[16]$} \\
\hline & & CMS & $0.90 \pm 0.05$ & $2.83 \pm 0.18$ & $0.792 \pm 0.024$ & {$[14]$} \\
\hline
\end{tabular}




\section{Competing Interests}

The author declares that there is no conflict of interests regarding the publication of this paper.

\section{Acknowledgments}

The author is grateful to Professor G. Alexander for useful discussions.

\section{References}

[1] W. Kittel, "Bose-Einstein correlations in $\mathrm{Z}$ fragmentation and other reactions," Acta Physica Polonica B, vol. 32, p. 3927, 2001.

[2] T. Csörgó, "Particle Interferometry from $40 \mathrm{MeV}$ to $40 \mathrm{TeV}$," Heavy Ion Physics, vol. 15, pp. 1-80, 2002.

[3] V. A. Okorokov, "Energy dependence of femtoscopy properties of pion source in nuclear collisions," https://arxiv.org/abs/1312 .4269 .

[4] V. A. Okorokov, "Geometry and space-time extent of pion emission region at FCC energies," Advances in High Energy Physics, vol. 2016, Article ID 5972709, 14 pages, 2016.

[5] A. Bialas, "Intermittency and the Hanbury-Brown-Twiss effect," Acta Physica Polonica B, vol. 23, pp. 561-567, 1992.

[6] A. Bialas, "Intermittency in high energy collisions," Nuclear Physics A, vol. 545, no. 1-2, pp. 285-296, 1992.

[7] P. Lévy, Theorie de l'Addition des Variables Aleatories, GauthierVilliers, Paris, France, 1937.

[8] V. V. Uchaikin, "Anomalous diffusion and fractional stable distributions," Journal of Experimental and Theoretical Physics, vol. 97, no. 4, pp. 810-825, 2003.

[9] V. V. Uchaikin, "Self-similar anomalous diffusion and Levystable laws," Physics-Uspekhi, vol. 46, no. 8, pp. 821-849, 2003.

[10] G. Goldhaber, S. Goldhaber, W. Lee, and A. Pais, "Influence of Bose-Einstein statistics on the antiproton-proton annihilation process," Physical Review, vol. 120, no. 1, pp. 300-312, 1960.

[11] D. H. Boal, C.-K. Gelbke, and B. K. Jennings, "Intensity interferometry in subatomic physics," Reviews of Modern Physics, vol. 62, no. 3, pp. 553-602, 1990.

[12] T. Csörgó, S. Hegyi, and W. A. Zajc, "Bose-Einstein correlations for Lévy stable source distributions," The European Physical Journal C, vol. 36, no. 1, pp. 67-78, 2004.

[13] C. Forbes, M. Evans, N. Hastings, and B. Peacock, Statistical Distributions, John Wiley \& Sons, New Jersey, NJ, USA, 2011.

[14] V. Khachatryan, A. M. Sirunyan, A. Tumasyan et al., "Measurement of Bose-Einstein correlations in pp collisions at $\sqrt{s}=0.9$ and 7 TeV,' Journal of High Energy Physics, vol. 2011, no. 5, article 29, 2011.

[15] V. Khachatryan, K. A. Bloom, S. Bose et al., "First measurement of bose-einstein correlations in proton-proton collisions at $\sqrt{s}=$ 0.9 and $2.36 \mathrm{TeV}$ at the LHC," Physical Review Letters, vol. 105, no. 3, Article ID 032001, 2010.

[16] R. Astaloš, Bose-Einstein correlations in $7 \mathrm{TeV}$ proton-proton collisions in the ATLAS experiment [Ph.D. thesis], Radboud University Nijmegen and Comenius University Bratislava, Nijmegen, The Netherlands, 2015.

[17] S. S. Padula, "Multidimensional analysis of Bose-Einstein correlations in pp collisions at 2.76 and $7 \mathrm{TeV}$ in CMS," in Proceedings of the Workshop on Particle Correlations and Femtoscopy (WPCF '14), T. Csörgó, M. Csanád, and T. Novák, Eds., eConf C140825.8, Scientific Publishing Services at SLAC, August 2014.
[18] C. Adler, Z. Ahammed, C. Allgower et al., "Pion interferometry of $\sqrt{s_{N N}}=130 \mathrm{GeV} \mathrm{Au}+\mathrm{Au}$ collisions at RHIC," Physical Review Letters, vol. 87, no. 8, Article ID 082301, 2001.

[19] B. I. Abelev, M. M. Aggarwal, Z. Ahammed et al., "Systematic measurements of identified particle spectra in $p p, d+A u$, and $\mathrm{Au}+\mathrm{Au}$ collisions at the STAR detector," Physical Review C, vol. 79, no. 3, Article ID 034909, 2009.

[20] L. J. Gutay, A. S. Hirsch, R. P. Scharenberg, B. K. Srivastava, and C. Pajares, "De-confinement in small systems: clustering of color sources in high multiplicity $\bar{p} p$ collisions at $\sqrt{s}=1.8 \mathrm{TeV}$," International Journal of Modern Physics E, vol. 24, no. 12, Article ID 1550101, 2015.

[21] G. Alexander and V. A. Okorokov, "The $\lambda$ energy dependence deduced from bose-einstein correlations of $\pi \pi$ pairs produced in pp collisions," Journal of Physics: Conference Series, vol. 675, no. 2, Article ID 022001, 2016.

[22] G. Aad, B. Abbott, J. Abdallah et al., "Two-particle Bose-Einstein correlations in $p p$ collisions at $\sqrt{s}=0.9$ and $7 \mathrm{TeV}$ measured with the ATLAS detector," The European Physical Journal C, vol. 75, article 466, 2015.

[23] M. M. Aggarwal, A. Agnihotri, Z. Ahammed et al., "Central $\mathrm{Pb}+\mathrm{Pb}$ collisions at $158 \mathrm{~A} \mathrm{GeV} / \mathrm{c}$ studied by $\pi^{-} \pi^{-}$interferometry," The European Physical Journal C, vol. 16, no. 3, pp. 445-451, 2000.

[24] R. Adler, T. Alhalel, A. Angelopoulos et al., "Bose-Einstein correlations inp $\bar{p} \mathrm{p}$ annihilations at rest," Zeitschrift für Physik C, vol. 63, no. 4, pp. 541-547, 1994.

[25] K. Aamodt, N. Abel, U. Abeysekara et al., "Two-pion BoseEinstein correlations in $p p$ collisions at $\sqrt{s}=900 \mathrm{GeV}$," Physical Review D, vol. 82, no. 5, Article ID 052001, 2010.

[26] F. Siklér, "Femtoscopy with identified hadrons in pp, pPb, and peripheral $\mathrm{PbPb}$ collisions in CMS," in Proceedings of the Workshop on Particle Correlations and Femtoscopy (WPCF '14), T. Csörgó, M. Csanád, and T. Novák, Eds., eConf C140825.8, Scientific Publishing Services at SLAC, August 2014.

[27] R. Albrecht, T. C. Awes, P. Beckmann et al., "Bose-einstein correlations in the target fragmentation region in $200 \mathrm{~A} \mathrm{GeV}^{16} \mathrm{O}+$ nucleus collisions," Zeitschrift für Physik C, vol. 53, no. 2, pp. 225-237, 1992.

[28] V. A. Okorokov, "Azimuthally integrated HBT parameters for charged pions in nucleus-nucleus interactions versus collision energy," Advances in High Energy Physics, vol. 2015, Article ID 790646, 13 pages, 2015.

[29] T. Åkesson, M. G. Albrow, S. Almehed et al., "Pion interferometry in jet events at the CERN Intersecting Storage Rings," Zeitschrift für Physik C, vol. 36, no. 4, pp. 517-526, 1987.

[30] I. Sýkora, "Measurements of Bose-Einstein correlations with the ATLAS detector," in Proceedings of the 23rd International Workshop on Deep-Inelastic Scattering and Related Subjects (DIS '15), p. 157, Dallas, Tex, USA, 2015.

[31] B. Abelev, J. Adamak, D. Adamová et al., "Freeze-out radii extracted from three-pion cumulants in $\mathrm{pp}, \mathrm{p}-\mathrm{Pb}$ and $\mathrm{Pb}-\mathrm{Pb}$ collisions at the LHC," Physics Letters B, vol. 739, pp. 139-151, 2014.

[32] J. Uribe, E. P. Hartouni, D. A. Jensen et al., "Pion-pion correlations at low relative momentum produced in $p-p$ collisions at $27.5 \mathrm{GeV} / c$," Physical Review D, vol. 49, no. 9, article 4373, 1994.

[33] J. L. Bailly, C. Caso, Y. Chiba et al., "Bose-Einstein correlations for pions produced in $p p$ collisions at $360 \mathrm{GeV} / c$," Zeitschrift für Physik C, vol. 43, no. 3, pp. 341-348, 1989. 
[34] M. Aguilar-Benitez, W. W. M. Allison, A. A. Batalov et al., "Bose-Einstein correlations in pp collisions at $400 \mathrm{GeV} / \mathrm{c}$," Zeitschrift für Physik C, vol. 54, no. 1, pp. 21-32, 1992.

[35] A. Breakstone, R. Campanini, H. B. Crawley et al., "Multiplicity dependence of the average transverse momentum and of the particle source size in $p-p$ interactions at $\sqrt{s}=62,44$ and 31 GeV," Zeitschrift für Physik C, vol. 33, no. 3, pp. 333-338, 1987.

[36] M. M. Aggarwal, Z. Ahammed, A. Alakhverdyants et al., "Pion femtoscopy in $p+p$ collisions at $\sqrt{s}=200 \mathrm{GeV}$," Physical Review C, vol. 83, no. 6, Article ID 064905, 2011.

[37] T. Alexopoulos, C. Allen, E. W. Anderson et al., "Study of source size in $p \bar{p}$ collisions at $\sqrt{s}=1.8 \mathrm{TeV}$ using pion interferometry," Physical Review D, vol. 48, no. 5, pp. 1931-1942, 1931.

[38] L. Lovás, The Bose-Einstein correlations in CDF II experiment [Ph.D. thesis], Comenius University in Bratislava, Bratislava, Slovakia, 2008.

[39] G. Aad, B. Abbott, J. Abdallah et al., "Charged-particle distributions in $\sqrt{s}=13 \mathrm{TeV} p p$ interactions measured with the ATLAS detector at the LHC," Physics Letters B, vol. 758, pp. 67-88, 2016.

[40] L. Ahle, Y. Akiba, K. Ashktorab et al., "System, centrality, and transverse mass dependence of two-pion correlation radii in heavy ion collisions at $11.6 \mathrm{~A}$ and $14.6 \mathrm{~A} \mathrm{GeV} / c$," Physical Review C, vol. 66, no. 5, Article ID 054906, 15 pages, 2002.

[41] H. Appelshäuser, J. Bächler, S. J. Bailey et al., "Hadronic expansion dynamics in central $\mathrm{Pb}+\mathrm{Pb}$ collisions at $158 \mathrm{GeV}$ per nucleon," The European Physical Journal C, vol. 2, no. 4, pp. 661-670, 1998.

[42] I. G. Bearden, H. Bøggild, J. G. Boissevain et al., "High energy $\mathrm{Pb}+\mathrm{Pb}$ collisions viewed by pion interferometry," Physical Review C, vol. 58, no. 3, pp. 1656-1665, 1998.

[43] K. Adcox, S. S. Adler, N. N. Ajitanand et al., "Transverse-mass dependence of two-pion correlations in $A u+A u$ collisions at $\sqrt{s_{\mathrm{NN}}}=130 \mathrm{GeV}$," Physical Review Letters, vol. 88, no. 19, Article ID 192302, 2002.

[44] A. Bamberger, D. Bangert, J. Bartke et al., "Probing the spacetime geometry of ultra-relativistic heavy-ion collisions," Physics Letters B, vol. 203, no. 3, pp. 320-326, 1988. 

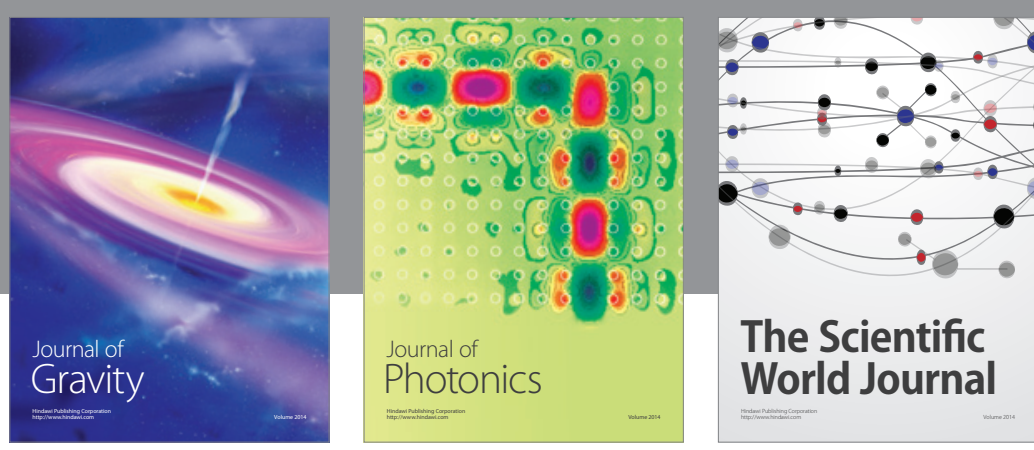

The Scientific World Journal
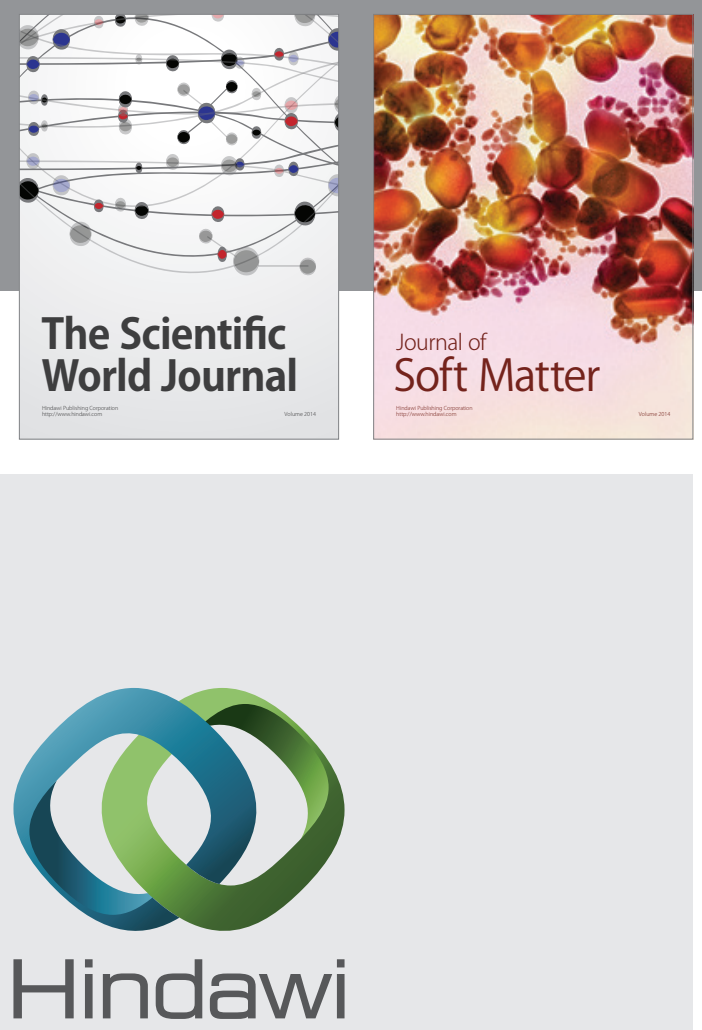

Submit your manuscripts at

https://www.hindawi.com
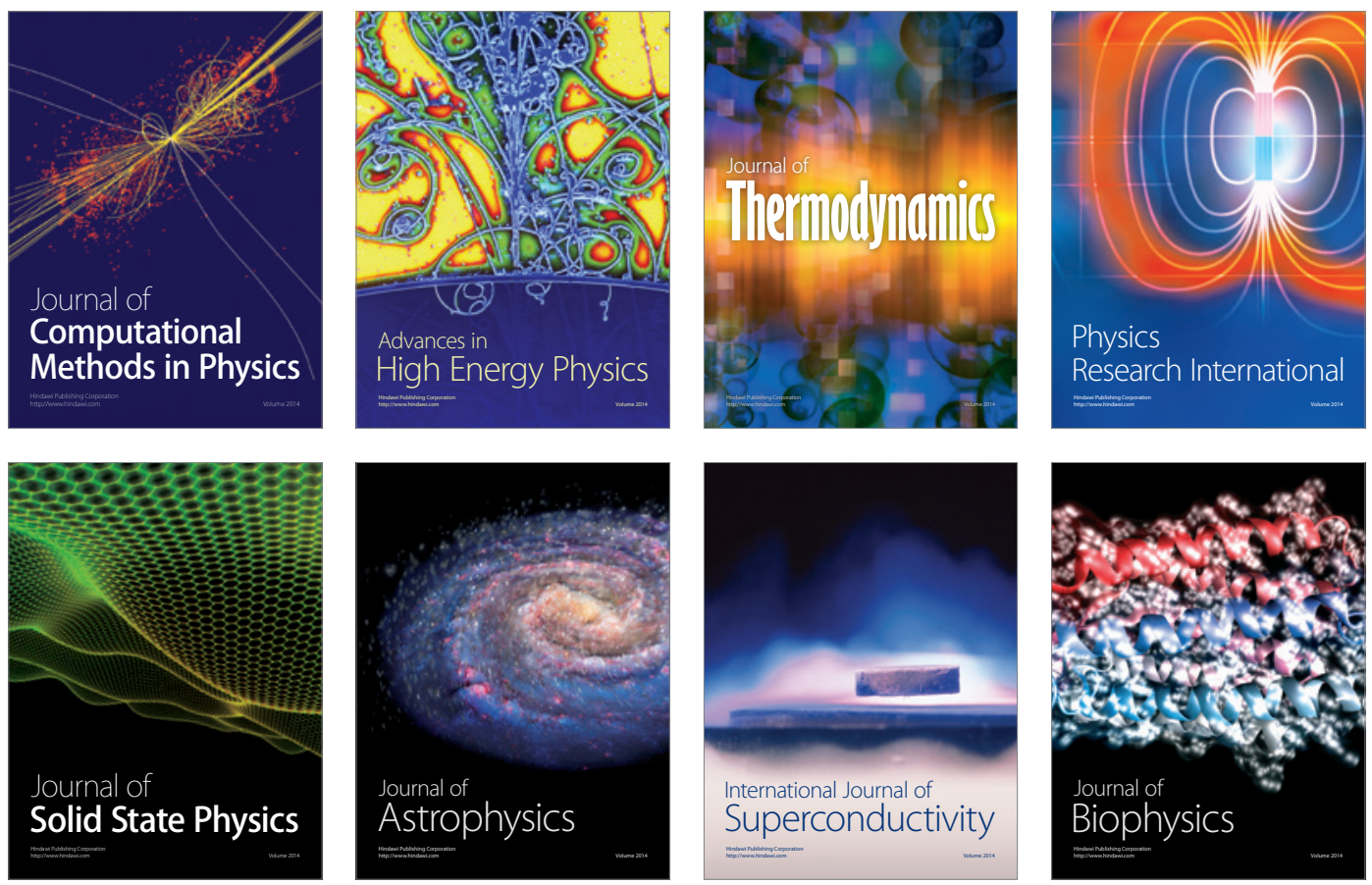
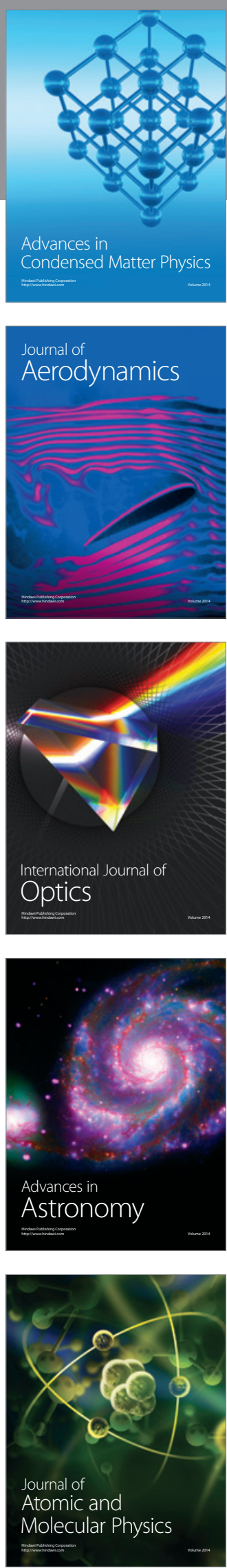